\title{
Etude du rayonnement solaire global et direct à Carpentras
}

\author{
L. M. Schwartz, E. Faure, R. Louat et A. Louat \\ Laboratoire d'Etudes Thermiques et Solaires, Université Claude Bernard Lyon I, \\ 43, Bd du 11 Novembre 1918, 69622 Villeurbanne Cedex, France
}

(Reçu le 12 novembre 1982, révisé le 17 mai 1983, accepté le 6 juin 1983)

\begin{abstract}
Résumé. - Dans cette étude, nous établissons des relations permettant le calcul, en fonction du temps, de l'éclairement énergétique global sur une surface horizontale et de l'éclairement énergétique direct sur une surface normale au rayonnement solaire, pour des journées types de temps clair à Carpentras en 1979.
\end{abstract}

\begin{abstract}
In this paper, we establish relations giving power of global radiation falling on a horizontal surface and of direct radiation on a normal surface, versus time, for typical clear days, in Carpentras (France), for the year 1979.
\end{abstract}

\section{Introduction.}

Dans une récente publication [1], nous avons établi une formule fournissant l'éclairement énergétique solaire global $G_{\mathrm{OH}}^{*}$ tombant sur une surface horizontale, par temps clair, en fonction de la durée $\tau$, en heures, comptées à partir de midi, temps solaire vrai :

$$
G_{\mathrm{OH}}^{*}=B(\cos \gamma \tau)^{\beta},
$$

et nous avons montré l'équivalence de cette formule avec la relation classique des cahiers de l'Afedes [2] :

$$
G_{\mathrm{OH}}^{*}=A(\sin h)^{\alpha},
$$

par l'établissement de relations entre les paramètres $A$, $\alpha$ et $B, \beta$, en nous appuyant sur des résultats de mesures effectuées à Mâcon par la Météorologie Nationale et à Lyon par nous-même.

En effet, si l'on pose

$$
\begin{aligned}
& \sin h=\sin \delta \sin \phi+\cos \delta \cos \phi \cos \omega \tau= \\
&=a+b \cos \omega \tau .
\end{aligned}
$$

On trouve :

$$
\begin{aligned}
& B=A(a+b)^{\alpha} \\
& \beta=\frac{b \alpha}{a+b} \cdot \frac{\omega^{2}}{\alpha^{2}} .
\end{aligned}
$$

La nomenclature des notations est donnée en fin de publication, afin de ne pas alourdir l'exposé.

Afin d'étendre cette étude au rayonnement direct, nous avons entrepris la mesure de ce rayonnement à
Lyon. Malheureusement, le nombre de journées de ciel bien clair y est relativement limité et nous n'avons pas encore pu recueillir suffisamment de données pour tester nos formules. Aussi, dans un premier temps, avons-nous utilisé les relevés d'irradiation globale horizontale et d'irradiation directe à Carpentras effectués par la Météorologie Nationale pour l'année 1979. Cette année a été très ensoleillée et on peut y relever une soixantaine de journées de ciel clair.

Nous avons admis qu'une journée était de ciel clair lorsque l'éclairement énergétique direct normal $I_{\mathrm{ON}}^{*}$ différait de moins de $10 \%$ de la valeur normalisée admise à cette date et à une heure déterminée pour le lieu en question, fournie par le tableau XVI, page 105 $\mathrm{du}$ Mémento d'Héliotechnique Mémosol $\left(\beta_{\mathrm{A}}=0,04\right)$ [3]. En outre, les valeurs relevées à des moments symétriques, de part et d'autre du midi solaire vrai, diffèrent aussi en général de moins de $20 \%$ (sauf au début et en fin de journée). Ces conditions sont remplies dans les tableaux I et II de cette publication.

Bien que le critère précédent soit assez restrictif, afin d'améliorer le calcul des coefficients définis dans la suite, nous n'avons pris en compte que les demijournées de ciel clair, soit le matin, soit le soir, en choisissant la partie qui fournissait l'énergie quotidienne maximale relevée sur les feuilles mensuelles de la station météo et nous avons, corrélativement, fait de même pour le rayonnement global horizontal.

\section{Rayonnement global sur le plan horizontal.}

Pour la soixantaine de journées sélectionnées, à peu près uniformément réparties sur l'année, nous avons 
déterminé les valeurs de $B$ et de $\beta$ à l'aide de la méthode des moindres carrés appliquée à la relation

$$
\ln G_{\mathrm{OH}}^{*}=\ln B+\beta \ln (\cos \gamma \tau) .
$$

Rappelons que la valeur de $\gamma$ est telle que $\cos \gamma \tau=0$ au lever et au coucher du soleil où $\tau= \pm \tau_{0}$. Alors $\gamma \tau_{0}=\pi / 2$; cette valeur étant mal définie, nous prendrons pour $\tau$ la moitié de la durée astronomique du jour. Si $d$ désigne le nombre de jours écoulés depuis l'équinoxe de printemps, la durée du jour $S S_{0}$ à Carpentras est approximativement fournie par la relation 2 :

$$
S S_{0}=12,0+3,3 \sin \frac{2 \pi d}{365} .
$$

Il en résulte que $\gamma=\pi / S S_{0}$.

Nous avons étudié les variations de $B$ et de $\beta$ en fonction de la date et cherché des corrélations avec la déclinaison $\delta$ ainsi qu'avec $\sin \delta$. La répartition des valeurs de $B$ en fonction de $\delta$ est plus régulière que celle qui est fonction de $\sin \delta$. Aussi avons-nous essayé une formule parabolique.

$$
B=a+b \delta+c \delta^{2},
$$

les coefficients $a, b$ et $c$ étant ajustés par régression multilinéaire. Nous avons trouvé :

$B=729,37+12,747 \delta-0,12883 \delta^{2} \quad\left(\mathrm{~W} \cdot \mathrm{m}^{-2}\right)$

où $\delta$ est exprimé en degrés.

Sur la figure 1, on représente les valeurs de $B$ calcu-

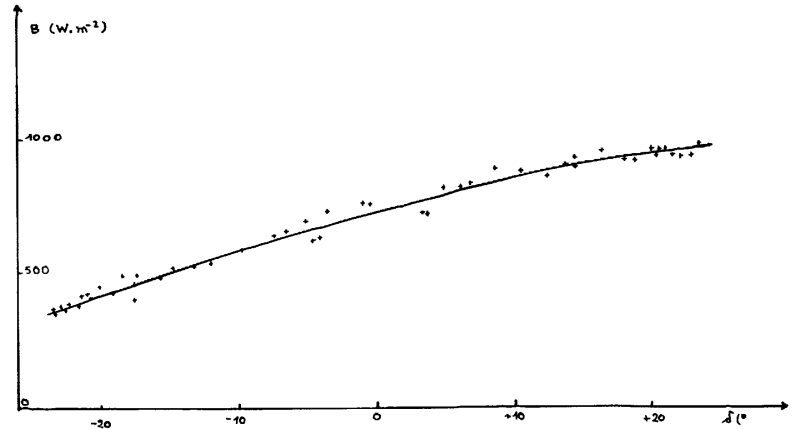

Fig. 1. - Rayonnement global. Variations de $B$ en fonction de $\delta$. + Points expérimentaux.

[Global radiation. Variation of $B$ versus $\delta$. + Experimental data.]

lées comme indiqué précédemment, ainsi que la fonction $B(\delta)$.

La figure 2 indique les valeurs de $\beta$. La fonction $\beta(\delta)$ peut être approchée par la relation linéaire en $\sin \delta$ :

$$
\beta=1,25041+0,27639 \sin \delta,
$$

les coefficients étant déterminés par la méthode des moindres carrés. Finalement, on peut écrire :

$$
\begin{aligned}
G_{\mathrm{OH}}^{*}=(729,37+ & \left.12,747 \delta-0,12883 \delta^{2}\right) \times \\
& \times(\cos \gamma \tau)^{(1,25041+0,27634 \sin \delta)}
\end{aligned}
$$

avec $\delta$ en degrés.

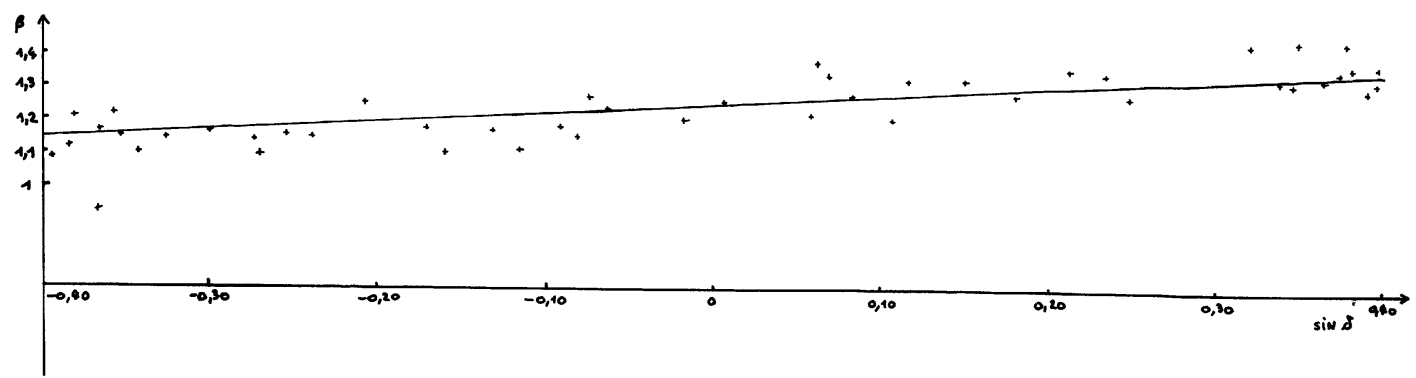

Fig. 2. - Variations de $\beta$ en fonction de $\sin \delta$. + Points expérimentaux.

[Variation of $\beta$ versus $\sin \delta$. + Experimental data.]

Le tableau I indique les valeurs de $G_{\mathrm{OH}}^{*}$ calculées à l'aide de la formule 5 pour quelques journées types, réparties sur toute l'année, choisies parmi toutes celles qui ont été sélectionnées pour les calculs et les compare aux valeurs expérimentales.

La vérification est bonne avec des erreurs inférieures à $8 \%$ sauf, en tout début et en fin de journée. La médiocre concordance pour la première et la dernière heure de la journée vient de ce que le soleil ne brille pas, en général, pendant tout cet intervalle de temps; il faudrait restreindre les intervalles de temps extrêmes à $1 / 2$ ou $1 / 4$ d'heure.

La figure 3 résume l'ensemble des ajustements : les courbes en trait plein fournissent l'éclairement éner- gétique correspondant au milieu de chaque échelon horaire, compté à partir de midi vrai et sont calculées à l'aide de la formule 5 ; les croix et les triangles représentent les valeurs mesurées.

Si l'on considère uniquement des valeurs de $\tau$ inférieures à 4 heures, la formule 5 peut se simplifier en :

$G_{\mathrm{OH}}^{*}=-22,04 \tau^{2}-14,92|\tau|+716,5+12,70 \delta$.

Dans le domaine de validité de cette formule simplifiée, les erreurs commises ne dépassent pas $10 \%$ en général.

Le tableau I fournit aussi l'énergie par unité de 
Tableau I. - La colonne 6 fournit les données brutes, relevées par la Météorologie Nationale à Carpentras, de l'énergie globale $G_{\mathrm{OH}}$ tombant sur le plan horizontal, exprimée en $\mathrm{J} . \mathrm{cm}^{-2}$, entre les temps $\tau-0,5$ et $\tau+0,5$, comptés en heures, à partir du midi solaire vrai. La colonne 7 indique l'éclairement énergétique moyen exprimé en $\mathrm{W} \cdot \mathrm{m}^{-2}$ au temps $\tau: G_{\mathrm{OH}}^{*}\left(\mathrm{~W} \cdot \mathrm{m}^{-2}\right)=\frac{G_{\mathrm{OH}}\left(\mathrm{J} \cdot \mathrm{cm}^{-2}\right)}{0,36}$. La colonne 8 donne les valeurs de $G_{\mathrm{OH}}^{*}$ calculées à l'aide de la formule 5. Dans les colonnes 11 et 12 figurent les irradiations $G_{\mathrm{OH}}$ exprimées en $\mathrm{Wh} . \mathrm{m}^{-2}$ cumulées sur toute la journée, mesurées et calculées à l'aide de la formule 6. Les colonnes 13, 14 et 15 indiquent respectivement les erreurs relatives, la moyenne des erreurs relatives et l'écart type pour les valeurs de la colonne 8 comparées à celles de la colonne 7.

[Column 6 gives the raw data measured by Météorologie Nationale in Carpentras, concerning the global energy $G_{\mathrm{OH}}\left(\mathrm{J} . \mathrm{cm}^{-2}\right)$ falling on a horizontal plane, between the time interval $\tau-0.5$ and $\tau+0.5(\tau$ in hours) elapsed from solar noon. Column 7 indicates irradiance $\left(\mathrm{W} . \mathrm{m}^{-2}\right)$ at time $\tau: G_{\mathrm{OH}}^{*}\left(\mathrm{~W} \cdot \mathrm{m}^{-2}\right)=\frac{G_{\mathrm{OH}}\left(\mathrm{J} . \mathrm{cm}^{-2}\right)}{0.36}$. Column 8 gives the values of $G_{\mathrm{OH}}^{*}$ calculated by formula 5 . In columns 11 and 12 , the energies $G_{\mathrm{OH}}\left(\mathrm{Wh} \mathrm{m}^{-2}\right)$ cumulated during a whole day, are measured and calculated by equation 6 . Columns 13, 14 and 15 give the relative errors, the mean value of the relative errors and the standard deviation for the values in column 8 , compared to those of column 7.]

\begin{tabular}{|c|c|c|c|c|c|c|c|c|c|c|c|c|c|c|}
\hline 1 & 2 & 3 & 4 & 5 & 6 & 7 & 8 & 9 & 10 & 11 & 12 & 13 & 14 & 15 \\
\hline Jour & $\delta\left(^{\circ}\right)$ & $\gamma$ & $\begin{array}{c}\text { heure } \\
\text { TSV }\end{array}$ & $t(h)$ & $\begin{array}{c}{ }_{\mathrm{O}}^{\mathrm{OH}} \\
\text { J.cm-2 } \\
\text { mesuré }\end{array}$ & $\begin{array}{l}\mathrm{G}_{\mathrm{OH}}^{\mathrm{x}} \\
\mathrm{W} \cdot \mathrm{m}^{-2} \\
\text { mesuré }\end{array}$ & 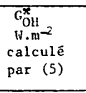 & $\stackrel{B}{11 \cdot m^{-2}}$ & $\begin{array}{l}\mathrm{c} \\
\mathrm{W} \\
\mathrm{m}\end{array}$ & 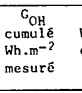 & 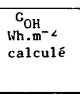 & 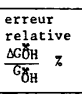 & $\begin{array}{l}\text { moyenne } \\
\text { des } \\
\text { erreurs } \\
\text { relatives }\end{array}$ & $\begin{array}{c}\text { ecart } \\
\text { type } \\
0 \\
w \cdot \mathrm{m}^{-2}\end{array}$ \\
\hline 16 Janv. & $-21,0$ & 0,34689 & $\begin{array}{l}07-08 \\
08-09 \\
09-10 \\
10-11 \\
11-12 \\
12-13 \\
13-14 \\
14-15 \\
15-16 \\
16-17\end{array}$ & $\begin{array}{l}4,5 \\
3,5 \\
2,5 \\
1,5 \\
0,5 \\
0,5 \\
1,5 \\
2,5 \\
3,5 \\
4,5\end{array}$ & $\begin{array}{r}6 \\
43 \\
89 \\
126 \\
145 \\
149 \\
127 \\
89 \\
42 \\
7\end{array}$ & $\begin{array}{r}16,6 \\
119,4 \\
247,2 \\
330,0 \\
402,8 \\
413,9 \\
352,8 \\
227,2 \\
116,7 \\
19,4\end{array}$ & $\begin{array}{r}- \\
120,8 \\
245,7 \\
344,7 \\
398,4 \\
398,6 \\
344,6 \\
244,4 \\
120,7 \\
21,9\end{array}$ & 420,5 & 1,219 & 2286,1 & 2275,1 & $\begin{array}{r}1,2 \\
0,6 \\
1,6 \\
1,0 \\
3,7 \\
2,4 \\
0,6 \\
3,5 \\
12,9\end{array}$ & 3,1 & 6,1 \\
\hline 06 PEvr. & $-15,9$ & 0,32123 & $\begin{array}{l}07-08 \\
07-09 \\
09-10 \\
10-11 \\
11-12 \\
12-13 \\
13-14 \\
14-15 \\
15-16 \\
16-17 \\
\end{array}$ & $\begin{array}{l}4,5 \\
3,5 \\
2,5 \\
1,5 \\
0,5 \\
0,5 \\
1,5 \\
2,5 \\
3,5 \\
4,5 \\
\end{array}$ & $\begin{array}{r}17 \\
67 \\
124 \\
1153 \\
173 \\
173 \\
154 \\
117 \\
49 \\
12 \\
\end{array}$ & $\begin{array}{r}47,2 \\
186,1 \\
344,4 \\
425,0 \\
480,6 \\
480,6 \\
427,8 \\
325,0 \\
136,1 \\
33,3 \\
\end{array}$ & $\begin{array}{r}42,7 \\
184,4 \\
322,5 \\
429,6 \\
487,7 \\
488,7 \\
429,6 \\
32,5 \\
184,5 \\
42,7 \\
\end{array}$ & $494,1 \quad 1$ & 1,175 & 2886,1 & 2922,2 & $\begin{array}{r}9,5 \\
0,9 \\
6,4 \\
1,1 \\
1,5 \\
1,5 \\
0,5 \\
0,4 \\
1,1 \\
35,5 \\
28,2\end{array}$ & 8,61 & 17,7 \\
\hline 08 Mars & $-5,2$ & 0,28150 & $\begin{array}{l}06-07 \\
07-08 \\
08-09 \\
09-10 \\
10-11 \\
11-12 \\
12-13 \\
13-14 \\
14-15 \\
15-16 \\
16-17 \\
17-18 \\
\end{array}$ & $\begin{array}{l}5,5 \\
4,5 \\
3,5 \\
2,5 \\
1,5 \\
0,5 \\
0,5 \\
1,5 \\
2,5 \\
3,5 \\
4,5 \\
5,5 \\
\end{array}$ & $\begin{array}{r}11 \\
59 \\
126 \\
180 \\
220 \\
239 \\
240 \\
215 \\
180 \\
124 \\
58 \\
10 \\
\end{array}$ & $\begin{array}{r}30,6 \\
163,9 \\
350,0 \\
500,0 \\
61,1,1 \\
663,9 \\
636,7 \\
599,7 \\
500,2 \\
34,0 \\
161,4 \\
27,8 \\
27,8 \\
\end{array}$ & $\begin{array}{r}35,9 \\
157,6 \\
324,4 \\
476,2 \\
590,4 \\
651,5 \\
651,5 \\
590,5 \\
476,4 \\
42,2 \\
15,4,4 \\
157,6 \\
35,9 \\
\end{array}$ & 659,6 & 1,225 & $\begin{array}{ll}5 & 4616,7\end{array}$ & 4406,4 & $\begin{array}{r}17 \\
3,8 \\
7,3 \\
4,8 \\
3,4 \\
1,9 \\
2,3 \\
1,1 \\
4,8 \\
5,8 \\
2,8 \\
29,2 \\
\end{array}$ & 7,0 & 11,5 \\
\hline 12 Avri1 & 8,7 & 0,23764 & $\begin{array}{l}05-06 \\
06-07 \\
07-08 \\
08-09 \\
09-10 \\
10-11 \\
11-12 \\
12-13 \\
13-14 \\
14-15 \\
15-16 \\
16-17 \\
17-18 \\
18-19 \\
\end{array}$ & $\begin{array}{l}6,5 \\
5,5 \\
4,5 \\
3,5 \\
2,5 \\
1,5 \\
0,5 \\
0,5 \\
1,5 \\
2,5 \\
3,5 \\
4,5 \\
5,5 \\
6,5 \\
\end{array}$ & $\begin{array}{r}7 \\
49 \\
117 \\
185 \\
241 \\
283 \\
305 \\
308 \\
288 \\
246 \\
191 \\
122 \\
52 \\
6 \\
\end{array}$ & $\begin{array}{r}19,4 \\
136,1 \\
325,0 \\
513,9 \\
669,4 \\
786,4 \\
847,2 \\
855,6 \\
800,0 \\
683,0 \\
530,6 \\
338,9 \\
144,4 \\
16,7 \\
\end{array}$ & $\begin{array}{r}17,3 \\
146,1 \\
322,0 \\
497,9 \\
650,7 \\
762,7 \\
821,9 \\
821,9 \\
762,7 \\
620,7 \\
497,9 \\
32,9 \\
146,1 \\
17,3 \\
\end{array}$ & 830,5 & 1,292 & 6666,7 & 6434,7 & $\begin{array}{r}11,0 \\
7,3 \\
1,0 \\
3,1 \\
2,8 \\
3,0 \\
3,0 \\
3,9 \\
4,7 \\
4,7 \\
6,2 \\
5,0 \\
1,2 \\
3,6 \\
\end{array}$ & 4,32 & 15,4 \\
\hline $21 \mathrm{Mai}$ & 20,4 & 0,21138 & $\begin{array}{l}04-05 \\
05-06 \\
06-07 \\
07-08 \\
08-09 \\
09-10 \\
10-11 \\
11-12 \\
12-13 \\
13-14 \\
14-15 \\
15-16 \\
16-17 \\
17-18 \\
18-19 \\
\end{array}$ & $\begin{array}{l}7,5 \\
6,5 \\
5,5 \\
4,5 \\
3,5 \\
2,5 \\
1,5 \\
0,5 \\
0,5 \\
1,5 \\
2,5 \\
3,5 \\
4,5 \\
5,5 \\
6,5 \\
\end{array}$ & $\begin{array}{r}2 \\
34 \\
96 \\
161 \\
224 \\
274 \\
310 \\
321 \\
328 \\
301 \\
271 \\
220 \\
157 \\
90 \\
32 \\
\end{array}$ & $\begin{array}{r}5,6 \\
94,4 \\
266,4 \\
447,2 \\
622,2 \\
76,1,1 \\
861,1 \\
891,1 \\
911,1 \\
81,1 \\
752,1 \\
75,8 \\
61,1,1 \\
436,1 \\
250,0 \\
88,9 \\
\end{array}$ & $\begin{array}{l}10 \overline{4}, 1 \\
268,9 \\
450,2 \\
622,2 \\
786,0 \\
873,0 \\
928,6 \\
928,6 \\
87,6 \\
768,4 \\
62,0 \\
450,2 \\
258,2 \\
104,1 \\
\end{array}$ & 935,8 & 1,347 & 7441,7 & 8033,4 & $\begin{array}{r}10,3 \\
1,2 \\
0,7 \\
0,0 \\
0,9 \\
1,4 \\
4,1 \\
1,9 \\
4,5 \\
2,0 \\
1,8 \\
3,2 \\
7,9 \\
17,1 \\
\end{array}$ & 4,1 & 10,5 \\
\hline 23 Juin & 23,5 & 0,20547 & $\begin{array}{l}04-05 \\
05-06 \\
06-07 \\
07-08 \\
08-09 \\
09-10 \\
10-11 \\
11-12 \\
12-13 \\
13-14 \\
14-15 \\
15-16 \\
16-17 \\
17-18 \\
18-19 \\
19-20\end{array}$ & $\begin{array}{l}7,5 \\
6,5 \\
5,5 \\
4,5 \\
3,5 \\
2,5 \\
1,5 \\
0,5 \\
0,5 \\
1,5 \\
2,5 \\
3,5 \\
4,5 \\
5,5 \\
6,5 \\
7,5\end{array}$ & $\begin{array}{r}7 \\
46 \\
108 \\
174 \\
235 \\
287 \\
323 \\
340 \\
341 \\
322 \\
285 \\
217 \\
156 \\
96 \\
40 \\
6\end{array}$ & 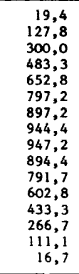 & $\begin{array}{l}13 \overline{-}, 2 \\
300,2 \\
309,2 \\
479,5 \\
649,4 \\
791,7 \\
894,6 \\
948,4 \\
948,4 \\
894,6 \\
791,7 \\
649,4 \\
479,5 \\
300,3 \\
132,2 \\
-\end{array}$ & 957,8 & 1,361 & 8286,1 & 8428,1 & $\begin{array}{r}5,0 \\
0,1 \\
0,8 \\
0,5 \\
0,7 \\
0,3 \\
0,4 \\
0,1 \\
0,0 \\
0,0 \\
7,7 \\
10,7 \\
12,6 \\
19,0\end{array}$ & 4,2 & 18,7 \\
\hline
\end{tabular}


Tableau I (suite).

\begin{tabular}{|c|c|c|c|c|c|c|c|c|c|c|c|c|c|c|}
\hline 1 & 2 & 3 & 4 & 5 & 6 & 7 & 8 & 9 & 10 & 11 & 12 & 13 & 14 & 15 \\
\hline jour & $\delta\left(^{\circ}\right)$ & y & $\begin{array}{c}\text { heure } \\
\text { TSV }\end{array}$ & $r(h)$ & $\begin{array}{c}C_{\mathrm{OH}} \\
\mathrm{J} . \mathrm{Cm}^{-2} \\
\text { mesure }\end{array}$ & 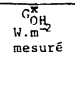 & 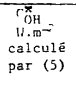 & $\underset{\mathrm{w} \cdot \mathrm{m}^{-2}}{\mathrm{~B}}$ & B & $\begin{array}{l}\mathrm{C}_{\mathrm{OOH}} \\
\text { cunulé } \\
\text { Wh..n-2 } \\
\text { mesuré }\end{array}$ & $\begin{array}{c}\mathrm{c}_{{ }_{\mathrm{OOH}}} \\
\text { wh.m-2 } \\
\text { calculé }\end{array}$ & 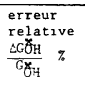 & $\begin{array}{l}\text { moyenne } \\
\text { des } \\
\text { erreurs } \\
\text { relat 2ves }\end{array}$ & $\begin{array}{c}\text { ecart } \\
\text { type } \\
\sigma \\
\mathrm{W} \cdot \mathrm{m}^{-2}\end{array}$ \\
\hline 15 Juill. & 21,5 & 0,20944 & $\begin{array}{l}04-05 \\
05-06 \\
06-07 \\
06-07 \\
07-08 \\
08-09 \\
09-10 \\
10-11 \\
11-12 \\
12-13 \\
13-14 \\
14-15 \\
15-16 \\
16-17 \\
17-18 \\
18-19 \\
19-20\end{array}$ & $\begin{array}{l}7,5 \\
6,5 \\
5,5 \\
4,5 \\
3,5 \\
2,5 \\
1,5 \\
0,5 \\
0,5 \\
1,5 \\
2,5 \\
3,5 \\
4,5 \\
5,5 \\
6,5 \\
7,5\end{array}$ & $\begin{array}{r}3 \\
37 \\
95 \\
161 \\
223 \\
275 \\
312 \\
332 \\
329 \\
311 \\
275 \\
225 \\
163 \\
97 \\
40 \\
4\end{array}$ & $\begin{array}{r}8,3 \\
102,3 \\
263,9 \\
447,9 \\
619,2 \\
763,4 \\
866,9 \\
922,7 \\
913,2 \\
863,9 \\
763,9 \\
625,9 \\
452,0 \\
269,8 \\
111,4 \\
11,1,1\end{array}$ & $\begin{array}{r}- \\
113,1 \\
279,5 \\
459,3 \\
630,4 \\
775,0 \\
879,4 \\
934,4 \\
934,1 \\
879,1 \\
775,4 \\
630,4 \\
459,3 \\
279,5 \\
113,1 \\
-\end{array}$ & 943,9 & 1,352 & 8005,6 & 8190,5 & $\begin{array}{r}10,0 \\
5,9 \\
2,7 \\
1,8 \\
1,5 \\
1,5 \\
1,3 \\
2,2 \\
1,8 \\
1,5 \\
0,9 \\
1,4 \\
3,7 \\
1,8\end{array}$ & 3,5 & 4,5 \\
\hline 12 Août & $-14,5$ & 0,22440 & $\begin{array}{l}05-06 \\
06-07 \\
07-08 \\
08-09 \\
09-10 \\
10-11 \\
11-12 \\
12-13 \\
13-14 \\
14-15 \\
15-16 \\
16-17 \\
17-18 \\
18-19\end{array}$ & $\begin{array}{l}6,5 \\
5,5 \\
4,5 \\
3,5 \\
2,5 \\
1,5 \\
0,5 \\
0,5 \\
1,5 \\
2,5 \\
3,5 \\
4,5 \\
5,5 \\
6,5\end{array}$ & $\begin{array}{r}19 \\
76 \\
142 \\
204 \\
257 \\
295 \\
312 \\
311 \\
291 \\
252 \\
201 \\
139 \\
73 \\
19\end{array}$ & $\begin{array}{r}52,8 \\
211,1 \\
394,4 \\
566,7 \\
713,9 \\
819,4 \\
866,7 \\
863,9 \\
808,3 \\
700,0 \\
558,3 \\
386,1 \\
202,8 \\
52,8\end{array}$ & $\begin{array}{r}51,1 \\
207,4 \\
386,9 \\
561,8 \\
71,6 \\
820,7 \\
888,0 \\
878,0 \\
880,7 \\
711,6 \\
561,8 \\
386,9 \\
207,4 \\
51,1\end{array}$ & 887,1 & 1,320 & 7197,2 & 7225,2 & $\begin{array}{l}3,2 \\
1,8 \\
1,9 \\
0,9 \\
0,3 \\
0,2 \\
1,3 \\
1,6 \\
1,5 \\
1,7 \\
0,6 \\
0,2 \\
2,3 \\
3,2\end{array}$ & 2,3 & 7,1 \\
\hline 22 Sept. & $-1,0$ & 0,26489 & $\begin{array}{l}06-07 \\
07-08 \\
07-09 \\
09-10 \\
09-10-11 \\
11-12 \\
112-13 \\
13-14 \\
14-15 \\
15-16 \\
16-17 \\
17-18 \\
18-19\end{array}$ & $\begin{array}{l}5,5 \\
4,5 \\
3,5 \\
2,5 \\
1,5 \\
0,5 \\
0,5 \\
1,5 \\
2,5 \\
3,5 \\
4,5 \\
5,5 \\
6,5\end{array}$ & $\begin{array}{r}23 \\
82 \\
146 \\
201 \\
239 \\
259 \\
256 \\
236 \\
195 \\
141 \\
77 \\
20 \\
0\end{array}$ & $\begin{array}{r}63,9 \\
227,8 \\
425,8 \\
558,6 \\
663,3 \\
719,9 \\
711,4,1 \\
655,1,6 \\
541,7 \\
391,7 \\
213,9 \\
55,6 \\
0\end{array}$ & $\begin{array}{r}207,4 \\
379,1 \\
532,7 \\
647,7 \\
708,2 \\
708,2 \\
647,2 \\
532,7 \\
379,1 \\
207,4 \\
47,6\end{array}$ & 716,5 & 1,246 & 5208,3 & 5041,4 & $\begin{array}{r}- \\
9,0 \\
6,5 \\
4,6 \\
2,6,5 \\
1,6 \\
0,4 \\
1,3 \\
1,7 \\
3,2 \\
3,0 \\
14,4\end{array}$ & 4,4 & 7,9 \\
\hline 19 oct. & $-11,4$ & 0,30208 & $\begin{array}{l}06-07 \\
07-08 \\
08-09 \\
09-90 \\
09-10 \\
10-11 \\
11-12 \\
12-13 \\
13-14 \\
14-15 \\
15-16 \\
16-17 \\
17-18\end{array}$ & $\begin{array}{l}5,5 \\
4,5 \\
3,5 \\
2,5 \\
1,5 \\
0,5 \\
0,5 \\
1,5 \\
2,5 \\
3,5 \\
4,5 \\
5,5\end{array}$ & $\begin{array}{r}3 \\
37 \\
101 \\
148 \\
186 \\
207 \\
208 \\
186 \\
148 \\
95 \\
38 \\
3\end{array}$ & $\begin{array}{r}8,3 \\
102,8 \\
280,6 \\
411,1 \\
516,7 \\
575,0 \\
577,8 \\
516,7 \\
411,1 \\
263,9 \\
105,6 \\
8,3\end{array}$ & $\begin{array}{r}- \\
87,4 \\
242,2 \\
387,9 \\
499,3 \\
555,9 \\
555,9 \\
499,9 \\
387,9 \\
242,2 \\
87,4 \\
-\end{array}$ & 567,3 & 1,196 & 3777,8 & 3548,1 & $\begin{array}{r}- \\
15,0 \\
13,7 \\
5,6 \\
3,4 \\
3,3 \\
3,8 \\
3,3 \\
5,6 \\
8,2 \\
17,2\end{array}$ & 7,9 & 6,5 \\
\hline 19 Nov. & $-20,3$ & 0,34328 & $\begin{array}{l}07-08 \\
08-09 \\
09-10 \\
10-11 \\
11-12 \\
12-13 \\
13-14 \\
14-15 \\
15-16 \\
16-17\end{array}$ & $\begin{array}{l}4,5 \\
3,5 \\
2,5 \\
1,5 \\
0,5 \\
0,5 \\
1,5 \\
2,5 \\
3,5 \\
4,5\end{array}$ & $\begin{array}{r}10 \\
50 \\
100 \\
135 \\
160 \\
155 \\
136 \\
98 \\
48 \\
8\end{array}$ & $\begin{array}{r}27,8 \\
138,9 \\
277,8 \\
375,0 \\
444,0 \\
430,6 \\
377,8 \\
272,2 \\
133,3 \\
22,2\end{array}$ & $\begin{array}{r}128,8 \\
255,7 \\
355,7 \\
410,4 \\
410,4 \\
355,7 \\
255,7 \\
128,8 \\
-8\end{array}$ & 417,5 & 1,155 & 2500,0 & 2323,5 & $\begin{array}{l}7,3 \\
8,0 \\
5,1 \\
7,6 \\
4,7 \\
5,8 \\
6,1 \\
3,4 \\
-\end{array}$ & 6,0 & 8,7 \\
\hline 18 Déc. & $-23,5$ & 0,36110 & $\begin{array}{l}07-08 \\
08-09 \\
09-10 \\
10-11 \\
11-12 \\
12-13 \\
13-14 \\
14-15 \\
15-16 \\
16-17\end{array}$ & $\begin{array}{l}4,5 \\
3,5 \\
2,5 \\
1,5 \\
0,5 \\
0,5 \\
1,5 \\
2,5 \\
3,5 \\
4,5\end{array}$ & $\begin{array}{r}3 \\
34 \\
76 \\
110 \\
131 \\
131 \\
112 \\
75 \\
32 \\
32\end{array}$ & $\begin{array}{r}8,3 \\
94,4 \\
211,1 \\
305,6 \\
363,9 \\
363,9 \\
311,1 \\
208,3 \\
88,9 \\
8,3\end{array}$ & $\begin{array}{r}-\overline{1,9} \\
208,1 \\
301,2 \\
352,6 \\
352,6 \\
301,2 \\
208,1 \\
91,9 \\
-\end{array}$ & 358,7 & 1,140 & 1963,9 & 1906,4 & $\begin{array}{l}- \\
2,6 \\
1,4 \\
1,4 \\
3,1 \\
3,1 \\
3,2 \\
0,1 \\
3,4\end{array}$ & 2,3 & 5,4 \\
\hline
\end{tabular}

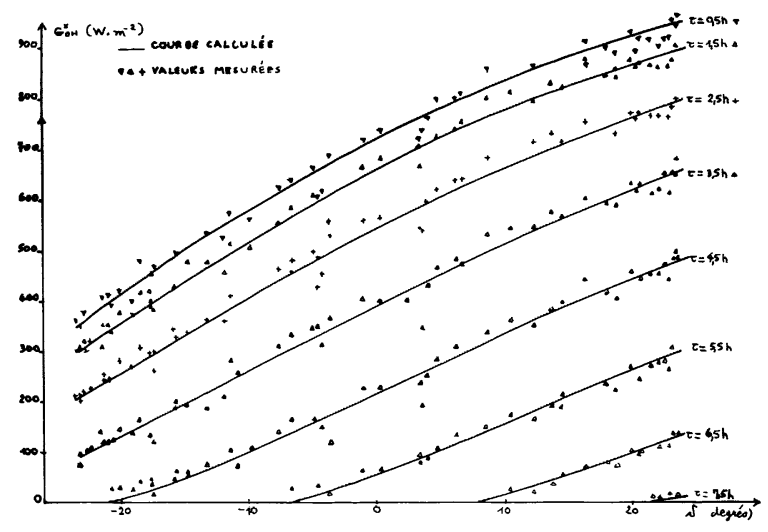

Fig. 3. - Valeurs mesurées et courbes calculées pour chaque valeur de $\tau$. courbe calculée, $\nabla \Delta+$ valeurs mesurées.

[Experimental values and curves calculated for each value of $\tau$. calculated curve, $\nabla \Delta+$ experimental data.] surface ou irradiation globale horizontale, cumulée sur une journée, calculée par une formule établie dans la publication 1 et comparée à la valeur mesurée :

$$
G_{\mathrm{OH}}=\frac{B}{\gamma} \sqrt{\pi} \frac{\Gamma\left(\frac{1+\beta}{2}\right)}{\Gamma\left(1+\frac{\beta}{2}\right)}
$$

où $\Gamma$ est la fonction eulérienne $\Gamma(x)=\int_{0}^{\infty} \mathrm{e}^{-t} t^{x-1} \mathrm{~d} t$.

\section{Rayonnement direct normal $I_{\mathrm{ON}}^{*}$.}

Nous avons procédé de la même manière que.pour le rayonnement global : nous avons admis une forme analogue, avec la même valeur de $\gamma$, et déterminé $\boldsymbol{B}^{\prime}$ 
et $\beta^{\prime}$ par la méthode des moindres carrés pour chaque journée type choisie :

$$
I_{\mathrm{ON}}^{*}=B^{\prime}(\cos \gamma \tau)^{\beta^{\prime}} .
$$

Nous avons approché les variations de $B^{\prime}$ et $\beta^{\prime}$ par des formules appropriées dont les coefficients ont été calculés par régression multilinéaire :

$$
B^{\prime}=918,25+1,71338 \delta-0,090659 \delta^{2} \quad\left(\mathrm{~W} \cdot \mathrm{m}^{-2}\right)
$$

$\beta^{\prime}=0,45754$.

La figure 4 fournit les valeurs de $B^{\prime}$ : la courbe en trait plein représente la fonction 7 ; la figure 5 traduit les

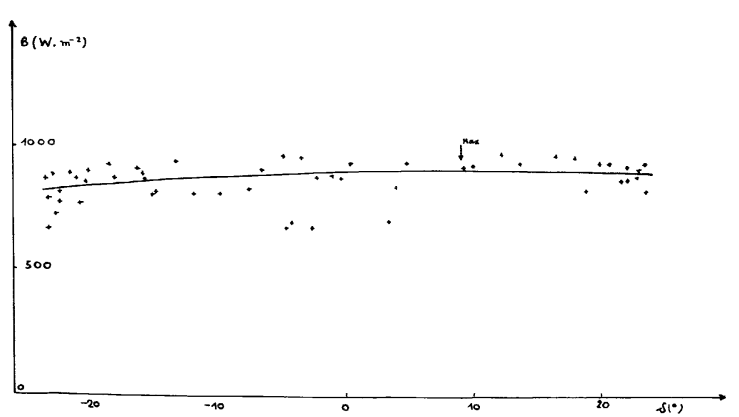

Fig. 4. - Rayonnement direct. Variations de $B^{\prime}$ en fonction de $\delta$. + Points expérimentaux.

[Direct radiation. Variation of $B^{\prime}$ versus $\delta$. + Experimental data.]

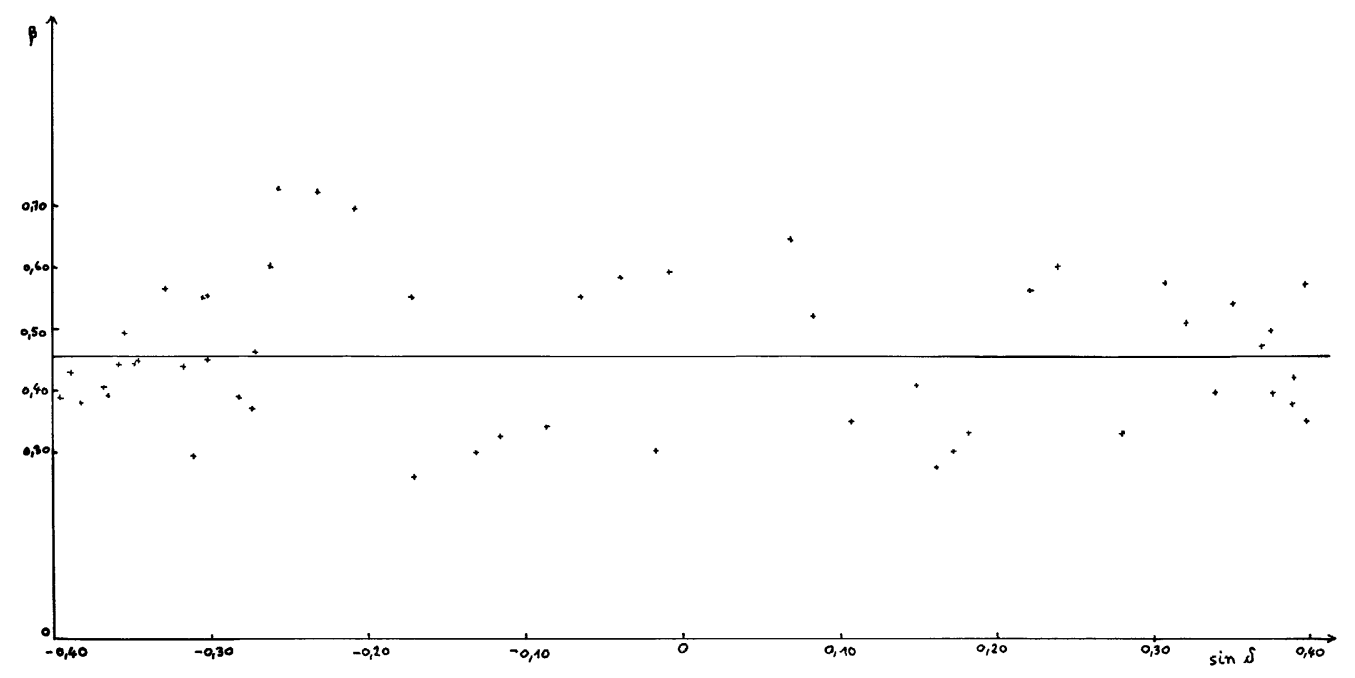

Fig. 5. - Rayonnement direct. Variations de $\beta$ en fonction de $\sin \delta$.

[Direct radiation. Variation of $\beta$ versus $\sin \delta$.]

valeurs expérimentales de $\beta^{\prime}$. On constate que la dispersion des valeurs de $\beta^{\prime}$ est relativement importante. Si l'on essaye d'approcher $\beta^{\prime}$ par une relation linéaire en $\delta$ ou $\sin \delta$, on obtient pratiquement la même valeur constante précédente (8).

D'où l'expression du rayonnement direct normal tout au long de l'année, par temps clair :

$$
\begin{array}{r}
I_{\mathrm{ON}}^{*}=\left(918,25+1,71338 \delta-0,090659 \delta^{2}\right) \times \\
\times(\cos \gamma \tau)^{0,4575}
\end{array}
$$

avec $\delta$ en degrés.

La figure 6 représente les courbes $I_{\mathrm{ON}}^{*}$ calculées, comme pour le rayonnement global, pour des valeurs demi-entières de $\tau$. La dispersion des points expérimentaux est beaucoup plus grande que pour le global et la vérification, surtout aux faibles valeurs de l'éclairement, est moins bonne.

Le tableau II compare les valeurs expérimentales aux valeurs calculées, pour les mêmes journées que le tableau I. Les erreurs ne dépassent guère $10 \%$ sauf en début et en fin de journée. En effet, le rayonnement direct est beaucoup plus sensible que le global au passage de légers nuages, même peu visibles à l'oil nu,

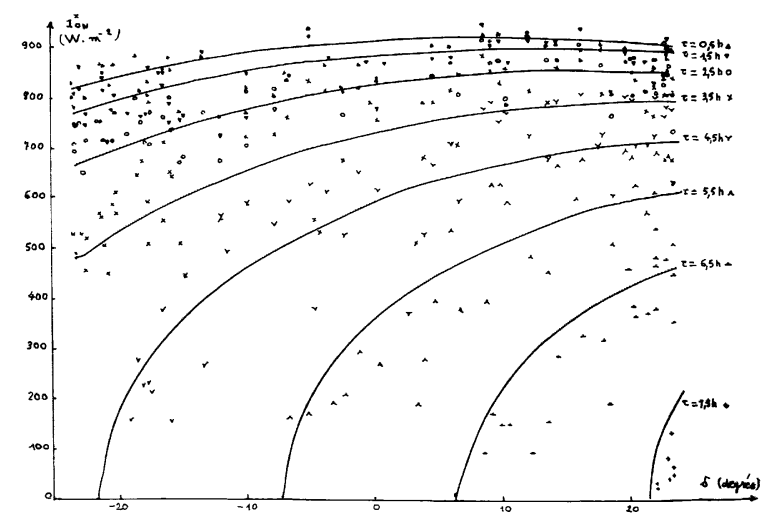

Fig. 6. - Rayonnement direct. Valeurs mesurées et courbes calculées pour chaque valeur de $\tau$. - courbe calculée, $\Delta \nabla \circ \times \vee \wedge \bumpeq+$ valeurs mesurées.

[Direct radiation. Experimental values and curves calculated for each value of $\tau$. - calculated curve, $\triangle \nabla O \times \vee \wedge\llcorner+$ experimental data.] 
Tableau II. - La colonne 6 fournit les données brutes, relevées par la Météorologie Nationale à Carpentras, de l'énergie directe $I_{\mathrm{ON}}$ tombant sur une surface normale au rayonnement solaire, exprimée en $\mathrm{J} . \mathrm{cm}^{-2}$, entre les temps $\tau-0,5$ et $\tau+0,5$, comptés en heures, à partir du midi solaire vrai. La colonne 7 indique l'éclairement énergétique moyen, exprimé en $\mathrm{W} . \mathrm{m}^{-2}$, au temps $\tau: I_{\mathrm{ON}}^{*}\left(\mathrm{~W} \cdot \mathrm{m}^{-2}\right)=\frac{I_{\mathrm{ON}}\left(\mathrm{J} . \mathrm{cm}^{-2}\right)}{0,36}$. La colonne 8 donne les valeurs de $I_{\mathrm{ON}}^{*}$ calculées à l'aide de la formule 9. Dans les colonnes 11 et 12 se trouvent les valeurs de l'irradiation $I_{\mathrm{ON}}$ cumulée sur une journée, mesurées et calculées par la formule 6. Les colonnes 13, 14 et 15 indiquent les erreurs relatives, la moyenne des erreurs relatives et l'écart type pour les valeurs de la colonne 8 comparées à celles de la colonne 7 . Enfin, la colonne 16 fournit $I_{\mathrm{ON}}^{*}$ calculé par l'équation 11 à partir de $G_{\mathrm{OH}}^{*}$ mesuré.

[Column 6 gives the raw data, measured by Météorologie Nationale in Carpentras, concerning the direct radiation $I_{\mathrm{ON}}\left(\mathrm{J} . \mathrm{cm}^{-2}\right)$ falling on a plane normal to the sunbeam, between the time interval $\tau-0.5$ and $\tau+0.5(\tau$ in hours) elapsed from solar noon. Column 7 indicates the average irradiance $\left(\mathrm{W} . \mathrm{m}^{-2}\right)$ at time $\tau: I_{\mathrm{OH}}^{*}\left(\mathrm{~W} \cdot \mathrm{m}^{-2}\right)=$ $\frac{I_{\mathrm{ON}}\left(\mathrm{J} . \mathrm{cm}^{-2}\right)}{0.36}$. Column 8 gives the values of $I_{\mathrm{ON}}^{*}$ calculated by formula 9 . In columns 11 and 12 , the energies $I_{\mathrm{ON}}$, cumulated during a whole day, are measured and calculated by equation 6 . Columns $13,14,15$ give the relative errors, the mean value of the relative errors and the standard deviation for the values in column 8 compared to those of column 7. Lastly, column 16 gives $I_{\mathrm{ON}}^{*}$ calculated by equation 11 from measured $G_{\mathrm{OH}}^{*}$.]

\begin{tabular}{|c|c|c|c|c|c|c|c|c|c|c|c|c|c|c|c|}
\hline 1 & 2 & 3 & 4 & 5 & 6 & 7 & 8 & 9 & 10 & 11 & 12 & 13 & 14 & 15 & 16 \\
\hline Jour & $\delta\left(^{\circ}\right)$ & r & $\begin{array}{c}\text { heure } \\
\text { TSV }\end{array}$ & $T(h)$ & $\begin{array}{l}{ }^{\mathrm{I}} \mathrm{ON}-2 \\
\text { J.cm } \\
\text { mesurế }\end{array}$ & $\begin{array}{l}{ }_{\mathrm{O}_{\mathrm{N}}}^{\mathrm{K}} \\
\mathrm{W}^{\mathrm{N}} \mathrm{m}^{-} \\
\text {mesuré }\end{array}$ & $\begin{array}{c}\mathrm{I}^{\mathrm{T}} \\
\text { WN- } \\
\text { W.m-2 } \\
\text { calculé } \\
\text { par (9) } \\
\text { par (9) }\end{array}$ & $\underset{\mathrm{W} \cdot \mathrm{m}^{-2}}{\mathrm{~B}}$ & B & 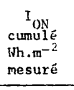 & $\begin{array}{l}\mathrm{I}_{\mathrm{nN}} \\
\mathrm{I}_{\mathrm{nN}}, \mathrm{m}^{-2} \\
\text { calculé }\end{array}$ & 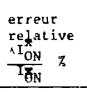 & $\begin{array}{l}\text { moyenne } \\
\text { des } \\
\text { erreurs } \\
\text { telatives }\end{array}$ & $\begin{array}{l}\text { Feart } \\
\text { cype } \\
\sigma \\
\text { w.m- }\end{array}$ & $\begin{array}{c}\text { IOX }_{\text {OX }}^{X} \\
\text { calculi } \\
\text { par (1 }\end{array}$ \\
\hline 16 Janv. & $-21,0$ & 0,34689 & $\begin{array}{l}07-08 \\
08-09 \\
09-10 \\
10-11 \\
11-12 \\
1-12 \\
13-13 \\
13-14 \\
14-15 \\
15-16 \\
16-17\end{array}$ & $\begin{array}{l}4,5 \\
3,5 \\
2,5 \\
1,5 \\
0,5 \\
0,5 \\
1,5 \\
2,5 \\
3,5 \\
4,5\end{array}$ & $\begin{array}{r}39 \\
203 \\
266 \\
289 \\
299 \\
311 \\
293 \\
258 \\
182 \\
40\end{array}$ & $\begin{array}{l}108,3 \\
563,9 \\
738,9 \\
80,98 \\
830,6 \\
883,9 \\
813,9 \\
716,7 \\
505,6 \\
111,1\end{array}$ & $\begin{array}{l}101,7 \\
520,9 \\
690,5 \\
798,6 \\
836,7 \\
836,7 \\
789,6 \\
690,5 \\
520,9 \\
101,7\end{array}$ & 842,3 & 0,457 & 6055,6 & 5929,6 & $\begin{array}{l}6,1 \\
7,6 \\
6,6 \\
1,6 \\
0,7 \\
3,1 \\
3,0 \\
3,7 \\
3,0 \\
8,5\end{array}$ & 4,4 & 20,1 & $\begin{array}{l}234,5 \\
51,6 \\
682,6 \\
782,1 \\
827,7 \\
827,4 \\
885,3 \\
785,1 \\
682,1 \\
507,0 \\
249,4\end{array}$ \\
\hline 6 Févr. & $-15,9$ & 0,32123 & $\begin{array}{l}07-08 \\
08-09 \\
09-10 \\
10-11 \\
11-12 \\
12-13 \\
13-14 \\
14-15 \\
15-16 \\
16-17\end{array}$ & $\begin{array}{l}4,5 \\
3,5 \\
2,5 \\
1,5 \\
0,5 \\
0,5 \\
1,5 \\
2,5 \\
3,5 \\
4,5\end{array}$ & $\begin{array}{r}56 \\
234 \\
288 \\
306 \\
317 \\
321 \\
311 \\
288 \\
102 \\
0\end{array}$ & $\begin{array}{c}155,6 \\
650,0 \\
800,0 \\
850,0 \\
880,6 \\
891,7 \\
863,9 \\
800,0 \\
283,0 \\
0,3\end{array}$ & $\begin{array}{c}591,6 \\
735,6 \\
821,7 \\
863,2 \\
863,2 \\
821,7 \\
735,1 \\
=\end{array}$ & 868,0 & 0,457 & 6175,0 & 6605,5 & $\begin{array}{l}\overline{9} \\
9,0 \\
8,1 \\
3,3 \\
2,0 \\
3,2 \\
4,9 \\
8,1 \\
-\end{array}$ & 5,5 & 21,1 & $\begin{array}{c}- \\
595,0 \\
755,6 \\
820,0 \\
880,0 \\
860,0 \\
822,0 \\
738,8 \\
-\end{array}$ \\
\hline 8 Mars & $-5,2$ & 0,28050 & $\begin{array}{l}06-07 \\
07-08 \\
08-09 \\
09-10 \\
10-11 \\
11-12 \\
12-13 \\
13-14 \\
14-15 \\
15-16 \\
16-17 \\
17-18\end{array}$ & $\begin{array}{l}5,5 \\
4,5 \\
3,5 \\
2,5 \\
1,5 \\
0,5 \\
0,5 \\
1,5 \\
2,5 \\
3,5 \\
4,5 \\
5,5\end{array}$ & $\begin{array}{r}62 \\
224 \\
295 \\
319 \\
331 \\
334 \\
338 \\
332 \\
324 \\
299 \\
225 \\
62\end{array}$ & $\begin{array}{l}172,2 \\
622,2 \\
819,4 \\
886,1 \\
919,4 \\
927,8 \\
938,9 \\
922,2 \\
900,0 \\
830,6 \\
625,0 \\
172,2\end{array}$ & $\begin{array}{l}177,2 \\
526,5 \\
693,9 \\
802,6 \\
80,6 \\
903,6 \\
903,6 \\
870,6 \\
80,6 \\
693,6 \\
593,9 \\
52,5 \\
177,2\end{array}$ & 908 & 0,457 & 8736,1 & 7893,5 & $\begin{array}{r}2,9 \\
15,4 \\
5,3 \\
9,4 \\
5,3 \\
2,6 \\
3,8 \\
5,6 \\
10,8 \\
16,5 \\
15,8 \\
2,9\end{array}$ & 8,9 & 47,9 & $\begin{array}{l}289,7 \\
542,1 \\
719,6 \\
82,6 \\
88,0 \\
813,0 \\
913,8 \\
915,2 \\
878,4 \\
82,4 \\
715,0 \\
538,2 \\
279,6 \\
279,5\end{array}$ \\
\hline 12 Avril & 8,7 & 0,23764 & $\begin{array}{l}05-06 \\
06-07 \\
07-08 \\
08-09 \\
09-10 \\
10-11 \\
11-12 \\
12-13 \\
13-14 \\
14-15 \\
15-16 \\
16-17 \\
17-18 \\
18-19 \\
\end{array}$ & $\begin{array}{l}6,5 \\
5,5 \\
4,5 \\
3,5 \\
2,5 \\
1,5 \\
0,5 \\
0,5 \\
1,5 \\
2,5 \\
3,5 \\
4,5 \\
5,5 \\
6,5\end{array}$ & $\begin{array}{r}14 \\
96 \\
239 \\
293 \\
313 \\
327 \\
335 \\
341 \\
341 \\
331 \\
319 \\
281 \\
195 \\
33\end{array}$ & $\begin{array}{r}38,9 \\
266,7 \\
663,9 \\
813,9 \\
869,4 \\
908,3 \\
930,6 \\
934,6 \\
947,2 \\
91,2 \\
88,4 \\
86,1 \\
760,6 \\
541,7 \\
91,7\end{array}$ & $\begin{array}{r}-\overline{-} \\
501,1 \\
662,8 \\
773,3 \\
850,1 \\
899,2 \\
923,3 \\
923,3 \\
899,2 \\
850,1 \\
773,3 \\
662,8 \\
501,1 \\
-\end{array}$ & 926,2 & 0,457 & 9605,6 & 9546,6 & $\begin{array}{r}8 \overline{8} \\
87,8 \\
0,2 \\
5,0 \\
2,2 \\
1,0 \\
0,8 \\
2,5 \\
5,1 \\
7,5 \\
71,7 \\
15,1 \\
7,5 \\
-\end{array}$ & 12,3 & 89,2 & $\begin{array}{l}48 \overline{-}, 3 \\
649,3 \\
775,0 \\
851,0 \\
90,0 \\
92,8 \\
92,9 \\
98,2 \\
906,4 \\
857,1 \\
783,1 \\
668,8 \\
498,6 \\
4,6\end{array}$ \\
\hline $21 \mathrm{Mai}$ & 20,4 & 0,21138 & $\begin{array}{l}04-05 \\
05-06 \\
06-07 \\
07-08 \\
08-09 \\
09-10 \\
10-11 \\
11-12 \\
12-13 \\
13-14 \\
14-15 \\
15-16 \\
16-17 \\
17-18 \\
18-19\end{array}$ & $\begin{array}{l}7,5 \\
6,5 \\
5,5 \\
4,5 \\
3,5 \\
2,5 \\
1,5 \\
0,5 \\
0,5 \\
1,5 \\
2,5 \\
3,5 \\
4,5 \\
6,5 \\
7,5\end{array}$ & $\begin{array}{r}0 \\
139 \\
247 \\
287 \\
307 \\
316 \\
316 \\
295 \\
303 \\
279 \\
282 \\
287 \\
259 \\
208 \\
105\end{array}$ & $\begin{array}{c}0 \\
386,1 \\
686,1 \\
797,2 \\
852,8 \\
877,8 \\
877,8 \\
819,8 \\
841,4 \\
775,7 \\
783,0 \\
797,2 \\
719,2 \\
557,4 \\
291,7\end{array}$ & $\begin{array}{r}-\bar{y} \\
434,3 \\
600,2 \\
714,1 \\
797,1 \\
856,1 \\
894,3 \\
913,2 \\
913,2 \\
894,3 \\
856,1 \\
797,1 \\
714,1 \\
600,2 \\
-\end{array}$ & 915,5 & 0,457 & 10100,0 & 10599,0 & $\begin{array}{r}- \\
12,5 \\
12,5 \\
10,4 \\
6,5 \\
2,5 \\
1,9 \\
11,4 \\
8,5 \\
15,4 \\
9,3 \\
0,0 \\
0,7 \\
3,9\end{array}$ & $?, 3$ & 65,6 & $\begin{array}{r}-7 \\
415,9 \\
590,4 \\
702,9 \\
785,8 \\
841,1 \\
816,9 \\
887,9 \\
893,7 \\
868,7 \\
838,0 \\
781,0 \\
697,0 \\
577,7 \\
\end{array}$ \\
\hline 23 Juin & 23,5 & 0,20547 & $\begin{array}{l}04-05 \\
05-06 \\
06-07 \\
06-07 \\
07-08 \\
08-09 \\
09-10 \\
10-11 \\
11-12 \\
12-12 \\
13-14 \\
14-15 \\
15-15 \\
16-16 \\
16-17 \\
17-18 \\
18-19 \\
19-20\end{array}$ & $\begin{array}{l}7,5 \\
6,5 \\
5,5 \\
4,5 \\
3,5 \\
2,5 \\
1,5 \\
0,5 \\
0,5 \\
1,5 \\
2,5 \\
3,5 \\
4,5 \\
5,5 \\
6,5 \\
7,5\end{array}$ & $\begin{array}{r}16 \\
161 \\
239 \\
280 \\
303 \\
312 \\
320 \\
321 \\
318 \\
322 \\
315 \\
244 \\
196 \\
158 \\
85 \\
7\end{array}$ & $\begin{array}{r}44,4 \\
447,2 \\
663,9 \\
777,8 \\
841,7 \\
866,7 \\
888,7 \\
891,9 \\
883,7 \\
894,3 \\
884,4 \\
675,0 \\
544,8 \\
438,4 \\
236,9 \\
19,1 \\
19,4\end{array}$ & $\begin{array}{c}467,0 \\
615,5 \\
720,6 \\
797,8 \\
853,8 \\
888,7 \\
906,4 \\
906,4 \\
888,4 \\
853,7 \\
797,0 \\
720,6 \\
615,5 \\
467,0\end{array}$ & 908,4 & 0,457 & 9991,7 & 10824,6 & $\begin{array}{r}- \\
4,4 \\
7,3 \\
7,4 \\
5,2 \\
1,6 \\
0,0 \\
1,6 \\
2,6 \\
0,6 \\
2,6 \\
17,5 \\
32,7 \\
40,4 \\
97,8\end{array}$ & 15,8 & 141,1 & $\begin{array}{l}45 \overline{7}, 7 \\
608,2 \\
712,9 \\
788,0 \\
842,0 \\
876,0 \\
89,0 \\
892,1 \\
892,0 \\
875,1 \\
840,2 \\
76,2 \\
687,3 \\
58,4 \\
436,8 \\
48,8\end{array}$ \\
\hline
\end{tabular}


Tableau II (suite).

\begin{tabular}{|c|c|c|c|c|c|c|c|c|c|c|c|c|c|c|c|}
\hline 1 & & 3 & 4 & 5 & 6 & 7 & 8 & 9 & 10 & 11 & 12 & 13 & 14 & 15 & 16 \\
\hline cour & $\left({ }^{\circ}\right)$ & he & $\begin{array}{l}\text { heure } \\
\text { TSV }\end{array}$ & $\tau(\mathrm{b})$ & 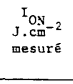 & 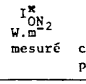 & $\begin{array}{c}\mathrm{I}_{\mathrm{ON}}^{\mathbf{X}} \\
\text { W.m } \\
\text { colcule } \\
\text { carcule } \\
\text { par }(9)\end{array}$ & $\stackrel{\mathrm{B}}{\mathrm{H} \cdot \mathrm{m}^{-2}}$ & $\begin{array}{ll}\mathrm{c} \\
\mathrm{in} \\
\mathrm{m}\end{array}$ & 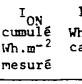 & 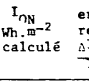 & $\begin{array}{l}\text { erreur } \\
\text { relative } \\
\frac{A_{0 N}{ }^{2}}{1 \delta_{N}} \\
\delta_{N}\end{array}$ & $\begin{array}{l}\text { moyenne } \\
\text { des } \\
\text { erreurs } \\
\text { relatives }\end{array}$ & $\begin{array}{l}\text { Écart } \\
\text { type } \\
c^{-} \\
\text {H.m. }^{-}\end{array}$ & $\begin{array}{l}\text { ION }_{\text {Ion }}^{\text {calcule }} \\
\text { par (11) }\end{array}$ \\
\hline 15 Juill. & 21,5 & 0,20944 & $\begin{array}{l}04-05 \\
05-06 \\
06-07 \\
06-07 \\
07-08 \\
08-09 \\
09-10 \\
10-11 \\
11-12 \\
12-12 \\
12-13 \\
13-14 \\
14-15 \\
15-16 \\
16-17 \\
17-18 \\
18-19 \\
19-20\end{array}$ & $\begin{array}{l}7,5 \\
6,5 \\
5,5 \\
4,5 \\
3,5 \\
2,5 \\
1,5 \\
0,5 \\
0,5 \\
1,5 \\
2,5 \\
2,5 \\
3,5 \\
4,5 \\
5,5 \\
6,5 \\
7,5\end{array}$ & $\begin{array}{r}4 \\
119 \\
205 \\
252 \\
278 \\
287 \\
289 \\
302 \\
294 \\
297 \\
294 \\
279 \\
255 \\
207 \\
133 \\
15\end{array}$ & $\begin{array}{l}11,1 \\
330,6 \\
569,4 \\
700,0 \\
712,0 \\
712,2 \\
979,2 \\
827,8 \\
838,9 \\
816,7 \\
825,0 \\
816,7 \\
715,7 \\
708,0 \\
575,3 \\
369,0 \\
41,4 \\
41,7\end{array}$ & $\begin{array}{l}- \\
445,5 \\
605,4 \\
716,3 \\
797,3 \\
855,1 \\
892,5 \\
910,9 \\
910,9 \\
892,5 \\
855,5 \\
797,1 \\
716,3 \\
605,4 \\
445,5\end{array}$ & 913,2 & 0,457 & 9775,0 & 10673,6 & $\begin{array}{r}- \\
34,8 \\
6,3 \\
2,3 \\
3,3 \\
7,3 \\
7,3 \\
8,8 \\
8,6 \\
11,5 \\
8,2 \\
4,7 \\
2,9 \\
1,1 \\
5,3 \\
20,3 \\
-\end{array}$ & 8,9 & 37,3 & $\begin{array}{l}427,2 \\
427,2 \\
586,3 \\
700,0 \\
780,9 \\
837,9 \\
874,9 \\
892,2 \\
889,6 \\
873,9 \\
837,29 \\
783,3 \\
702,9 \\
590,4 \\
438,5 \\
-7\end{array}$ \\
\hline 12 Aolt & 14,5 & 0,22440 & $\begin{array}{l}05-06 \\
06-07 \\
07-08 \\
08-09 \\
09-10 \\
10-11 \\
11-12 \\
12-13 \\
13-14 \\
14-15 \\
15-16 \\
16-17 \\
17-18 \\
18-19 \\
\end{array}$ & $\begin{array}{l}6,5 \\
5,5 \\
4,5 \\
3,5 \\
2,5 \\
1,5 \\
0,5 \\
0,5 \\
1,5 \\
2,5 \\
3,5 \\
4,5 \\
5,5 \\
6,5 \\
\end{array}$ & $\begin{array}{l}103 \\
241 \\
287 \\
310 \\
317 \\
327 \\
329 \\
322 \\
322 \\
313 \\
297 \\
277 \\
225 \\
103 \\
\end{array}$ & $\begin{array}{l}286,1 \\
669,4 \\
797,2 \\
861,2 \\
880,6 \\
980,6 \\
913,3 \\
894,9 \\
894,4 \\
869,4 \\
892,4 \\
769,0 \\
69,4 \\
625,0 \\
286,1 \\
\end{array}$ & $\begin{array}{l}339,7 \\
556,9 \\
692,5 \\
788,7 \\
856,4 \\
90,4 \\
921,0 \\
921,4 \\
90,4 \\
856,0 \\
78,4 \\
692,7 \\
552,5 \\
339,9 \\
399,7\end{array}$ & 924,0 & 0,457 & 10480,0 & 0 10076,6 & $\begin{array}{r}18,7 \\
20,2 \\
13,1 \\
8,4 \\
2,7 \\
0,9 \\
0,8 \\
3,0 \\
0,6 \\
1,5 \\
4,4 \\
10,0 \\
10,9 \\
18,7 \\
\end{array}$ & 9,1 & 50,9 & $\begin{array}{l}343,9 \\
555,2 \\
689,1 \\
781,1 \\
846,0 \\
887,3 \\
904,7 \\
903,7 \\
883,2 \\
840,3 \\
777,1 \\
684,1 \\
547,6 \\
343,9\end{array}$ \\
\hline 22 Sept. & $-1,0$ & 0,26489 & $\begin{array}{l}06-07 \\
07-08 \\
08-09 \\
09-10 \\
10-11 \\
11-12 \\
12-13 \\
13-14 \\
14-15 \\
15-16 \\
16-17 \\
17-18\end{array}$ & $\begin{array}{l}5,5 \\
4,5 \\
3,5 \\
2,5 \\
1,5 \\
0,5 \\
0,5 \\
1,5 \\
2,5 \\
3,5 \\
4,5 \\
5,5\end{array}$ & $\begin{array}{l}105 \\
234 \\
281 \\
302 \\
313 \\
312 \\
300 \\
301 \\
284 \\
258 \\
210 \\
86\end{array}$ & $\begin{array}{l}291,7 \\
650,0 \\
780,6 \\
838,9 \\
869,4 \\
866,7 \\
83,7 \\
836,1 \\
788,1 \\
716,7 \\
58,7 \\
238,9 \\
238,9\end{array}$ & $\begin{array}{l}339,2 \\
581,6 \\
725,7 \\
822,2 \\
883,1 \\
912,8 \\
912,8 \\
883,1 \\
82,1 \\
725,2 \\
581,6 \\
339,2 \\
39\end{array}$ & 916,4 & 0,457 & 8294,4 & 8447,5 & $\begin{array}{r}16,3 \\
10,5 \\
7,0 \\
2,0 \\
1,6 \\
5,3 \\
9,5 \\
5,6 \\
4,2 \\
1,3 \\
0,3 \\
42,0\end{array}$ & 8,8 & 50,0 & $\begin{array}{l}377,7 \\
602,5 \\
744,7 \\
837,5 \\
892,5 \\
919,3 \\
915,4 \\
888,4 \\
828,4 \\
735,3 \\
588,7 \\
358,9\end{array}$ \\
\hline 19 octob. & $-11,4$ & 0,30208 & $\begin{array}{l}06-07 \\
07-08 \\
08-09 \\
09-10 \\
10-11 \\
11-12 \\
12-13 \\
13-14 \\
14-15 \\
15-16 \\
16-17 \\
17-18 \\
\end{array}$ & $\begin{array}{l}5,5 \\
4,5 \\
3,5 \\
2,5 \\
1,5 \\
0,5 \\
0,5 \\
1,5 \\
2,5 \\
3,5 \\
4,5 \\
5,5 \\
\end{array}$ & $\begin{array}{r}13 \\
153 \\
253 \\
268 \\
292 \\
307 \\
312 \\
305 \\
291 \\
260 \\
176 \\
21 \\
\end{array}$ & $\begin{array}{r}36,1 \\
425,0 \\
702,8 \\
744,4 \\
811,1 \\
852,8 \\
86,8,7 \\
847,2 \\
808,3 \\
722,2 \\
48,2 \\
58,3 \\
\end{array}$ & $\begin{array}{r}- \\
437,4 \\
645,1 \\
772,3 \\
850,4 \\
888,1 \\
888,1 \\
850,4 \\
772,3 \\
645,1 \\
437,4 \\
-\end{array}$ & 886,9 & 0,457 & 7363,9 & 7173,9 & $\begin{array}{c}- \\
2,9 \\
8,2 \\
3,7 \\
4,8 \\
4,1 \\
2,5 \\
0,4 \\
4,5 \\
10,7 \\
10,5 \\
-\end{array}$ & 5,2 & 43,1 & $\begin{array}{c}-\overline{7}, 1 \\
674,1 \\
789,2 \\
86,3,4 \\
897,3 \\
898,9 \\
861,4 \\
789,3 \\
666,3 \\
469,7 \\
-\end{array}$ \\
\hline 19 Nov. & $-20,3$ & 0,34328 & $\begin{array}{r}07-08 \\
08-09 \\
09-10 \\
10-11 \\
111-12 \\
12-13 \\
13-14 \\
14-15 \\
15-16 \\
16-17 \\
\end{array}$ & $\begin{array}{l}4,5 \\
3,5 \\
2,5 \\
1,5 \\
0,5 \\
0,5 \\
1,5 \\
2,5 \\
3,5 \\
4,5\end{array}$ & $\begin{array}{r}56 \\
234 \\
288 \\
306 \\
317 \\
321 \\
311 \\
288 \\
102 \\
0\end{array}$ & $\begin{array}{c}155,6 \\
655,0 \\
800,0 \\
850,0 \\
880,6 \\
891,7 \\
863,9 \\
800,0 \\
283,0 \\
0\end{array}$ & $\begin{array}{l}591,6 \\
735,6 \\
82,1,7 \\
863,2 \\
863,2 \\
821,7 \\
733,1 \\
591,6 \\
-\end{array}$ & 868,0 & 0,457 & 6157,0 & 6605,5 & $\begin{array}{l}-7 \\
9,0 \\
8,1 \\
3,3 \\
2,0 \\
3,2 \\
4,9 \\
8,1 \\
10,9 \\
-9\end{array}$ & 18,5 & 125,8 & $\begin{array}{c}- \\
541,4 \\
711,6 \\
801,0 \\
856,2 \\
845,9 \\
803,3 \\
705,9 \\
532,7\end{array}$ \\
\hline 18 Déc. & $-23,5$ & 0,36110 & $\begin{array}{l}07-08 \\
08-09 \\
09-10 \\
10-11 \\
11-12 \\
12-13 \\
13-14 \\
14-14 \\
15-15 \\
15-16 \\
16-17\end{array}$ & $\begin{array}{l}4,5 \\
3,5 \\
2,5 \\
1,5 \\
0,5 \\
0,5 \\
1,5 \\
2,5 \\
3,5 \\
4,5\end{array}$ & $\begin{array}{r}18 \\
190 \\
267 \\
292 \\
300 \\
301 \\
290 \\
250 \\
162 \\
18\end{array}$ & $\begin{array}{r}50,0 \\
527,8 \\
741,7 \\
81,7,1 \\
833,3 \\
836,1 \\
805,6 \\
64,6 \\
450,4 \\
50,0 \\
50,0\end{array}$ & $\begin{array}{l}-7 \\
479,2 \\
665,2 \\
771,5 \\
821,8 \\
821,8 \\
771,5 \\
665,5 \\
479,2 \\
-2\end{array}$ & 827,9 & 0,457 & 5800,0 & 5602,2 & $\begin{array}{r}-, 2 \\
9,2 \\
10,3 \\
4,9 \\
1,4 \\
1,7 \\
4,2 \\
4,2 \\
6,5 \\
-9\end{array}$ & 5,3 & 30,9 & $\begin{array}{r}-17,0 \\
41,0 \\
659,3 \\
752,5 \\
806,8 \\
806,8 \\
757,9 \\
645,8 \\
459,8\end{array}$ \\
\hline
\end{tabular}

surtout le matin et le soir. Il en résulte, sur une journée entière, une répartition de l'énergie qui est moins symétrique par rapport au midi vrai que pour le global.

Pour des valeurs de $\tau$ comprises entre 0,5 et 2,5 , les courbes $I_{\mathrm{ON}}^{*}$ de la figure 6 présentent un maximum. Pour $\tau=0,5$ heure, ce maximum se produit pour $\delta=9,5^{\circ}$ et vaut $925 \mathrm{~W} \cdot \mathrm{m}^{-2}$. Pour $\delta=23,5^{\circ}$, $I_{\mathrm{ON}}^{*}=906 \cdot \mathrm{W} \cdot \mathrm{m}^{-2}$. La différence n'est que de $2 \%$ et tombe dans les limites d'erreur des mesures pyrhéliométriques. Cette précision est insuffisante pour mettre ce maximum expérimentalement en évidence.

Le tableau II fournit aussi l'irradiation $I_{\mathrm{ON}}$ cumulée sur une surface constamment normale au rayonnement solaire à l'aide de la relation 6 . Là encore, l'accord entre le calcul et l'expérience est en général bon.

\section{Relations entre le rayonnement global et le rayonne- ment direct.}

On peut éliminer $\cos \gamma \tau$ entre les équations 5 et 9.
On trouve :

$$
\begin{array}{r}
I_{\mathrm{ON}}^{*}=\left\{\frac{918,25+1,71338 \delta-0,090659 \delta^{2}}{729,37+12,747 \delta-0,12883 \delta^{2}}\right\} \times \\
\times G_{\mathrm{OH}}^{*}\left(\frac{0,45754}{1,25041+0,27639 \sin \delta}\right) .
\end{array}
$$

L'intérêt de cette formule est que, par temps clair, tout au moins, on pourrait songer à évaluer $I_{\mathrm{ON}}^{*}$ connaissant seulement $G_{\mathrm{OH}}^{*}$, valeur mesurée au pyranomètre, et se passer partiellement de l'appareil encombrant qu'est le pyrhéliomètre. Cependant, cette formule est à manier avec précaution car la présence de nuages hauts ou d'aérosols peut agir simultanément, mais de façon inverse, sur les deux composants du global sans le modifier lui-même sensiblement. Ainsi une faible diminution du global peut cacher une forte atténuation du direct. On peut sensiblement simplifier cette expression en effectuant un développement limité 
de la fraction rationnelle de gauche jusqu'au deuxième ordre en $\delta$ ainsi qu'un développement limité au premier ordre de l'exposant. On obtient :

$$
\begin{array}{r}
I_{\mathrm{ON}}^{*}=\left(82,29510+0,30218 \delta+0,0028486 \delta^{2}\right) \times \\
\times G_{\mathrm{OH}}^{*(0,366-0,0014 \sin \delta) .}
\end{array}
$$

La comparaison entre les valeurs mesurées de $I_{\mathrm{ON}}^{*}$ et celles calculées par la formule 11 montre que, sauf au tout début et en fin de journée, la vérification est relativement satisfaisante dans la mesure où la distribution de l'énergie directe est suffisamment régulière au long de la journée considérée, ce qui rejoint la remarque précédente au sujet de la validité de cette formule.

\section{Rayonnement diffus sur le plan horizontal et facteur de trouble.}

On sait que le rayonnement direct est très sensible à l'état de l'atmosphère en particulier à la présence de vapeur d'eau et d'aérosols. Le rapport entre les éclairements énergétiques directs normaux, reçus sur terre et dans l'espace extra-terrestre fait intervenir un facteur, appelé facteur de trouble de Linke, pour la détermination duquel existe un grand nombre de formules. L'une des meilleures semble être celle de Kasten :

$$
T_{\mathrm{L}}=-\ln \left(\frac{J_{\mathrm{N}}^{*}}{I_{\mathrm{ON}}^{*}}\right) \cdot(0,9+9,4 \sin h)
$$

où $J_{\mathbf{N}}^{*}$ représente l'éclairement énergétique direct du rayonnement extra-terrestre sur une surface normale à ce rayonnement.

Nous avons d'abord comparé les valeurs des facteurs de trouble obtenus par la formule de Kasten à celles que nous a fournies la station radiométrique de Carpentras pour les journées types retenues (Tableau III).

Tableau III. - Les colonnes 6, 7 et 8 indiquent les mesures du Centre radiométrique de Carpentras, les colonnes 10 et 11 le rayonnement diffus reç, par temps clair sur un plan horizontal, calculé respectivement par la formule classique et la relation 15.

[Columns 6, 7 and 8 give the values measured by Centre Radiométrique de Carpentras, columns 10 and 11 ,

\begin{tabular}{|c|c|c|c|c|c|c|c|c|c|c|c|c|c|}
\hline 1 & 2 & 3 & 4 & 5 & 6 & 7 & 8 & 9 & 10 & 11 & 12 & 13 & 14 \\
\hline Date & $\begin{array}{l}\text { heure } \\
\text { TSV }\end{array}$ & $h\left(0^{\circ}\right)$ & $\delta\left(^{\circ}\right)$ & $\begin{array}{l}\mathrm{J}_{\mathrm{N}}^{\mathrm{x}} \\
\left(\mathrm{II}, \mathrm{m}^{-2}\right)\end{array}$ & 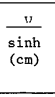 & ${ }^{B}{ }_{A}$ & $\begin{array}{l}\mathrm{T}_{\mathrm{L}} \\
\text { Mêtééo }\end{array}$ & $\begin{array}{l}\mathrm{r}_{\mathrm{L}} \\
\text { RASTEN }\end{array}$ & $\begin{array}{l}\mathrm{D}_{\mathrm{OH}}^{\mathrm{x}}= \\
G_{\mathrm{OH}}^{*}-I_{\mathrm{ON}}^{\boldsymbol{k}} \sinh \\
\left(\mathrm{W} \cdot \mathrm{m}^{-2}\right)\end{array}$ & $\begin{array}{l}\mathrm{D}_{\mathrm{OH}}^{\mathrm{x}} \\
\text { calculé } \\
\text { par (15) } \\
\left(\mathrm{W} \cdot \mathrm{m}^{-2}\right) \\
\end{array}$ & 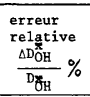 & $\begin{array}{l}\text { moyenne } \\
\text { des } \\
\text { erreurs } \\
\text { relatives } \\
\quad \%\end{array}$ & 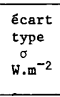 \\
\hline 16 Janv. & $\begin{array}{l}10 \mathrm{~h} 30 \\
12 \mathrm{~h} 30 \\
14 \mathrm{~h} 30\end{array}$ & $\begin{array}{l}21,81 \\
24,64 \\
16,49\end{array}$ & $-21,1$ & 1412 & $\begin{array}{l}1,69 \\
1,29 \\
1,67\end{array}$ & $\begin{array}{l}0,048 \\
0,046 \\
0,052\end{array}$ & $\begin{array}{l}2,38 \\
2,36 \\
2,39\end{array}$ & $\begin{array}{l}2,48 \\
2,22 \\
2,41\end{array}$ & $\begin{array}{l}51,7 \\
53,7 \\
43,8\end{array}$ & $\begin{array}{r}53,9 \\
42,4 \\
50,8\end{array}$ & $\begin{array}{r}4,3 \\
21,0 \\
16,0\end{array}$ & & \\
\hline 6 Févr. & $\begin{array}{l}10 \mathrm{~h} 30 \\
12 \mathrm{~h} 30 \\
14 \mathrm{~h} 30\end{array}$ & $\begin{array}{l}26,67 \\
29,71 \\
21,01\end{array}$ & $-15,9$ & 1405 & $\begin{array}{l}5,71 \\
3,55 \\
3,83\end{array}$ & $\begin{array}{l}0,028 \\
0,028 \\
0,033\end{array}$ & $\begin{array}{l}2,44 \\
2,50 \\
2,43\end{array}$ & $\begin{array}{l}2,57 \\
2,53 \\
2,40\end{array}$ & $\begin{array}{l}43,5 \\
38,7 \\
38,2\end{array}$ & $\begin{array}{l}57,8 \\
56,1 \\
50,3\end{array}$ & $\begin{array}{l}32,9 \\
45,0 \\
31,7\end{array}$ & & \\
\hline 8 Mars & $\begin{array}{l}10 \mathrm{~h} 30 \\
12 \mathrm{~h} 30 \\
14 \mathrm{~h} 30\end{array}$ & $\begin{array}{l}36,79 \\
40,34 \\
30,36\end{array}$ & $-5,2$ & 1389 & $\begin{array}{l}2,33 \\
1,59 \\
1,74\end{array}$ & $\begin{array}{l}0,040 \\
0,046 \\
0,036\end{array}$ & $\begin{array}{l}2,43 \\
2,61 \\
2,35\end{array}$ & $\begin{array}{l}2,69 \\
2,73 \\
2,45\end{array}$ & $\begin{array}{l}60,5 \\
58,9 \\
45,1\end{array}$ & $\begin{array}{l}63,1 \\
64,9 \\
52,5\end{array}$ & $\begin{array}{c}4,3 \\
10,2 \\
16,4\end{array}$ & & \\
\hline 12 Avril & $\begin{array}{l}10 \mathrm{hh} 30 \\
12 \mathrm{~h} 30 \\
14 \mathrm{~h} 30\end{array}$ & $\begin{array}{l}49,64 \\
54,10 \\
42,01\end{array}$ & 8,7 & 1361 & $\begin{array}{l}3,19 \\
2,13 \\
1,80\end{array}$ & $\begin{array}{l}0,065 \\
0,051 \\
0,043\end{array}$ & $\begin{array}{l}3,01 \\
2,90 \\
2,53\end{array}$ & $\begin{array}{l}3,26 \\
3,09 \\
2,82\end{array}$ & $\begin{array}{l}94,0 \\
88,3 \\
67,7\end{array}$ & $\begin{array}{l}88,3 \\
80,8 \\
68,9\end{array}$ & $\begin{array}{l}6,1 \\
8,5 \\
1,8\end{array}$ & & \\
\hline $21 \mathrm{Mai}$ & $\begin{array}{l}10 \mathrm{~h} 30 \\
12 \mathrm{~h} 30 \\
14 \mathrm{~h} 30\end{array}$ & $\begin{array}{l}59,89 \\
65,69 \\
50,99\end{array}$ & 20,4 & 1334 & $\begin{array}{l}2,75 \\
2,21 \\
2,96\end{array}$ & $\begin{array}{l}0,055 \\
0,094 \\
0,089\end{array}$ & $\begin{array}{l}3,17 \\
3,83 \\
3,76\end{array}$ & $\begin{array}{l}3,78 \\
4,35 \\
4,37\end{array}$ & $\begin{array}{l}101,7 \\
144,0 \\
144,1\end{array}$ & $\begin{array}{l}111,2 \\
136,4 \\
137,3\end{array}$ & $\begin{array}{l}9,3 \\
5,3 \\
4,7\end{array}$ & & \\
\hline 23 Juin & $\begin{array}{l}10 \mathrm{~h} 30 \\
12 \mathrm{~h} 30 \\
14 \mathrm{~h} 30\end{array}$ & $\begin{array}{l}62,43 \\
68,60 \\
53,16\end{array}$ & 23,5 & 1322 & $\begin{array}{l}3,30 \\
2,78 \\
-\end{array}$ & $\begin{array}{l}0,062 \\
0,064 \\
-\end{array}$ & $\begin{array}{l}3,27 \\
3,54 \\
-\end{array}$ & $\begin{array}{l}3,66 \\
3,89 \\
3,48\end{array}$ & $\begin{array}{r}109,2 \\
124,8 \\
91,4\end{array}$ & $\begin{array}{r}106,0 \\
116,1 \\
98,0\end{array}$ & $\begin{array}{l}2,9 \\
7,0 \\
7,2\end{array}$ & 14,0 & 12,1 \\
\hline 15 Juil. & $\begin{array}{l}10 \mathrm{hb} 3 \theta \\
12 \mathrm{~h} 30 \\
14 \mathrm{~h} 30\end{array}$ & $\begin{array}{l}60,80 \\
66,66 \\
51,77\end{array}$ & 21,5 & 1323 & $\begin{array}{l}3,53 \\
3,33 \\
3,64\end{array}$ & $\begin{array}{l}0,106 \\
0,110 \\
0,097\end{array}$ & $\begin{array}{l}3,93 \\
4,26 \\
3,78\end{array}$ & $\begin{array}{l}4,27 \\
4,60 \\
4,00\end{array}$ & $\begin{array}{l}144,1 \\
114,0 \\
122,4\end{array}$ & $\begin{array}{l}132,9 \\
147,4 \\
121,0\end{array}$ & $\begin{array}{r}7,8 \\
29,3 \\
1,1\end{array}$ & & \\
\hline 12 Août & $\begin{array}{l}10 \mathrm{~h} 30 \\
12 \mathrm{~h} 30 \\
1 / 4 \mathrm{~h} 30\end{array}$ & $\begin{array}{l}54,82 \\
59,81 \\
46,59\end{array}$ & 14,5 & 1330 & $\begin{array}{l}4,17 \\
3,01 \\
3,88\end{array}$ & $\begin{array}{l}0,040 \\
0,044 \\
0,043\end{array}$ & $\begin{array}{l}2,92 \\
3,30 \\
3,05\end{array}$ & $\begin{array}{l}3,27 \\
3,58 \\
3,29\end{array}$ & $\begin{array}{l}77,0 \\
90,8 \\
68,4\end{array}$ & $\begin{array}{r}88,7 \\
102,4 \\
89,5\end{array}$ & $\begin{array}{l}15,2 \\
12,8 \\
31,0\end{array}$ & & \\
\hline 22 Sept. & $\begin{array}{l}10 \mathrm{~h} 30 \\
12 \mathrm{~h} 30 \\
14 \mathrm{~h} 30\end{array}$ & $\begin{array}{l}40,72 \\
44,50 \\
33,95\end{array}$ & $-1,0$ & 1358 & $\begin{array}{l}2,94 \\
2,14 \\
2,87\end{array}$ & $\begin{array}{l}0,050 \\
0,065 \\
0,089\end{array}$ & $\begin{array}{l}2,85 \\
3,18 \\
3,31\end{array}$ & $\begin{array}{l}3,14 \\
3,66 \\
3,34\end{array}$ & $\begin{array}{r}96,7 \\
127,0 \\
101,0\end{array}$ & $\begin{array}{r}83,0 \\
106,0 \\
91,8\end{array}$ & $\begin{array}{r}14,2 \\
16,5 \\
9,1\end{array}$ & & \\
\hline 19 oct. & $\begin{array}{l}10 \mathrm{~h} 30 \\
12 \mathrm{~h} 30 \\
14 \mathrm{~h} 30\end{array}$ & $\begin{array}{l}30,94 \\
34,18 \\
24,97\end{array}$ & $-11,4$ & 1378 & $\begin{array}{l}3,84 \\
3,57 \\
4,53\end{array}$ & $\begin{array}{l}0,058 \\
0,049 \\
0,044\end{array}$ & $\begin{array}{l}2,91 \\
2,83 \\
2,69\end{array}$ & $\begin{array}{l}3,04 \\
2,87 \\
2,60\end{array}$ & $\begin{array}{l}99,7 \\
90,9 \\
69,9\end{array}$ & $\begin{array}{l}78,6 \\
71,1 \\
59,2\end{array}$ & $\begin{array}{l}21,2 \\
21,8 \\
15,3\end{array}$ & & \\
\hline 19 Nov. & $\begin{array}{l}10 \mathrm{~h} 30 \\
12 \mathrm{~h} 30 \\
14 \mathrm{~h} 30\end{array}$ & $\begin{array}{l}22,48 \\
25,33 \\
17,11\end{array}$ & $-20,3$ & 1400 & $\begin{array}{l}4,12 \\
2,38 \\
2,66\end{array}$ & $\begin{array}{l}0,041 \\
0,051 \\
0,043\end{array}$ & $\begin{array}{l}2,24 \\
2,22 \\
2,05\end{array}$ & $\begin{array}{l}2,24 \\
2,22 \\
2,05\end{array}$ & $\begin{array}{l}50,0 \\
49,1 \\
36,8\end{array}$ & $\begin{array}{l}43,3 \\
42,4 \\
34,9\end{array}$ & $\begin{array}{r}13,4 \\
13,6 \\
5,2\end{array}$ & & \\
\hline 18 Dếc. & $\begin{array}{l}10 \mathrm{~h} 30 \\
12 \mathrm{~h} 30 \\
14 \mathrm{~h} 30\end{array}$ & $\begin{array}{l}19,42 \\
22,15 \\
14,26\end{array}$ & $-23,5$ & 1412 & $\begin{array}{l}3,47 \\
2,59 \\
3,11\end{array}$ & $\begin{array}{l}0,019 \\
0,026 \\
0,024\end{array}$ & $\begin{array}{l}2,23 \\
2,41 \\
2,42\end{array}$ & $\begin{array}{l}2,13 \\
2,41 \\
2,42\end{array}$ & $\begin{array}{l}35,9 \\
54,1 \\
44,4\end{array}$ & $\begin{array}{l}42,8 \\
50,8 \\
51,2\end{array}$ & $\begin{array}{r}19,2 \\
6,1 \\
15,3\end{array}$ & & \\
\hline
\end{tabular}
the diffuse radiation falling, by clear weather, on a horizontal plane, calculated respectively by the classical formula and relation 15.]

Les heures TSV indiquées dans le tableau correspondent approximativement aux instants des trois mesures journalières de la hauteur d'eau condensable $w$ et du coefficient de trouble d'Angström $\beta_{\mathrm{A}}$. En général, les résultats ne diffèrent pas de plus de $20 \%$, la correspondance étant d'ailleurs meilleure en hiver qu'en été à cause, probablement de l'influence de la hauteur d'eau condensable qui ne figure pas dans la formule de Kasten.

Avec les valeurs qui nous ont été indiquées pour $w$ et $\beta_{\mathrm{A}}$ nous avons essayé de vérifier une formule du type :

$$
T_{\mathrm{L}}=a+b \beta_{\mathrm{A}}+c \ln w .
$$


Nous avons trouvé par régression multilinéaire :

$$
T_{\mathrm{L}}=1,70+18,7 \beta_{\mathrm{A}}+0,73 \ln w .
$$

Les coefficients de cette relation sont assez voisins de ceux donnés par Perrin de Brichambaut et Vauge [4].

Nous avons ensuite recherché une corrélation entre le rayonnement diffus horizontal et le facteur de trouble de Linke.

On sait que :

$$
D_{\mathrm{OH}}^{*}=G_{\mathrm{OH}}^{*}-I_{\mathrm{ON}}^{*} \sin h
$$

varie peu avec le temps autour du midi solaire vrai.
En première approximation, on peut assimiler les courbes $D_{\mathrm{H}}$ en fonction du temps à des droites horizontales (cf. courbes, p. 142 du livre cité en référence 4). Pour obtenir les valeurs de $D_{\mathrm{H}}$, il suffit donc de poser $\tau=0$, alors :

$$
G_{\mathrm{OH}}^{*} \simeq 716,5+12,70 \delta
$$

$\sin h=a+b \cos \omega \tau=a+b=\sin \delta \sin \phi+\cos \delta \cos \phi$ et

$$
I_{\mathrm{OH}}^{*}=J_{\mathrm{N}}^{*} \exp \left(-\frac{T_{\mathrm{L}}}{0,9+9,4(\sin \delta \sin \phi+\cos \delta \cos \phi)}\right) .
$$

Finalement, avec les valeurs précédentes, on obtient :

$$
D_{\mathrm{OH}}^{*}=716,5+12,70 \delta-J_{\mathrm{N}}^{*} \exp \left(-\frac{T_{\mathrm{L}}}{0,9+9,4(\sin \delta \sin \phi+\cos \delta \cos \phi)}\right)(\sin \delta \sin \phi+\cos \delta \cos \phi) .
$$

Au cours de l'année $J_{\mathrm{N}}^{*}$ est constant à $3,4 \%$ près, d'autre part, on a toujours $2<T_{\mathrm{L}}<4,5$. Pour Carpentras, $\sin \phi \simeq \cos \phi \simeq 0,7$, donc, avec la valeur moyenne de $J_{\mathrm{N}}^{*}=1370 \mathrm{~W} \cdot \mathrm{m}^{-2}$ :

$$
D_{\mathrm{OH}}^{*} \simeq 716,5+12,70 \delta-959(\sin \delta+\cos \delta) \cdot \exp \left(-\frac{T_{\mathrm{L}}}{0,9+6,58(\sin \delta+\cos \delta)}\right) .
$$

Si l'on trace, pour différentes valeurs de $T_{\mathrm{L}}$, les courbes représentant les variations de la quantité

$$
X=959(\sin \delta+\cos \delta) \exp \left(-\frac{T_{\mathrm{L}}}{0,9+6,58(\sin \delta+\cos \delta)}\right) .
$$

On remarque que celles-ci sont voisines de droites parallèles et quasi équidistantes pour des valeurs de $T_{\mathrm{L}}$ variant en progression arithmétique (Fig. 7). Il en résulte que $X$ est une fonction linéaire en $\delta$ et $T_{\mathrm{L}}$. On trouve :

$$
X \simeq 750+14 \delta-50 T_{\mathrm{L}} .
$$

D'où : $D_{\mathrm{OH}}^{*} \simeq 717+12,7 \delta-750-14 \delta+50 T_{\mathrm{L}}$.

Il en résulte que $D_{\mathrm{OH}}^{*}$ ne dépend pratiquement pas de $\delta$ et peut s'écrire grossièrement :

$$
D_{\mathrm{OH}}^{*} \simeq 50 T_{\mathrm{L}}-33\left(\mathrm{~W} \cdot \mathrm{m}^{-2}\right) .
$$

Cette formule remarquablement simple nécessite une

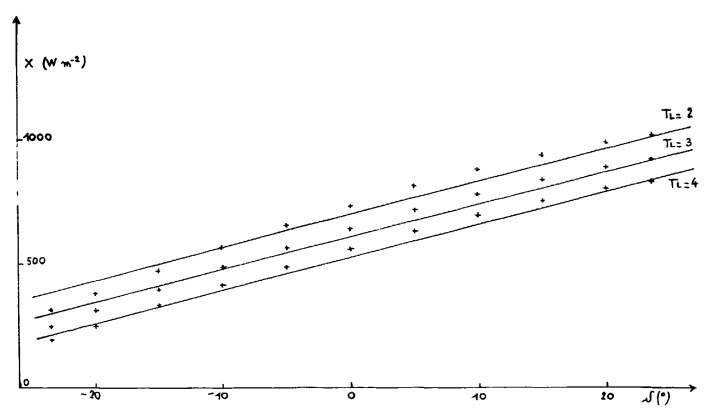

Fig. 7. - Variations de $X$ en fonction de $\delta$. + Points calculés.

[Variations of $X$ versus $\delta .+$ Calculated data.] REVUE DE PHYSIQUE APPLIQUÉE. - T. 18, No 10 , OCTOBRE 1983 vérification expérimentale. Si l'on représente (Fig. 8) les valeurs de $D_{\mathrm{OH}}^{*}$ calculées par la formule $D_{\mathrm{OH}}^{*}=$ $G_{\mathrm{OH}}^{*}-I_{\mathrm{OH}}^{*} \sin h$ en fonction du facteur de trouble $T_{\mathrm{L}}$ déterminé par la formule Kasten, on constate que les

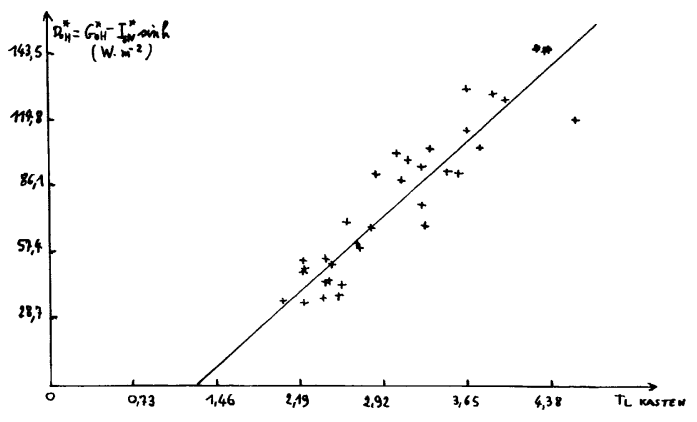

Fig. 8. - Relation entre $D_{\mathrm{OH}}^{*}$ et $T_{\mathrm{L}}$.

[Relation between $D_{\mathrm{OH}}^{*}$ and $T_{\mathrm{L}}$.]

points représentatifs sont assez voisins d'une droite dont l'équation, déterminée par la méthode des moindres carrés, est :

$$
D_{\mathrm{OH}}^{*}=44,14 T_{\mathrm{L}}-55,6\left(\mathrm{~W} \cdot \mathrm{m}^{-2}\right) .
$$

Les coefficients de cette droite ont des valeurs effectivement voisines de celles de la relation 14 .

Le tableau III fournit, pour toutes les journées 
types retenues, et à différentes heures de ces journées, le coefficient $T_{\mathrm{L}}$ déterminé par le service météo de Carpentras, le coefficient $T_{\mathrm{L}}$ de Kasten, les valeurs de $D_{\mathrm{OH}}^{*}$ calculées à partir de $G_{\mathrm{OH}}^{*}$ et $I_{\mathrm{ON}}^{*}$ mesurés, les valeurs de $D_{\mathrm{OH}}^{*}$ calculées à partir de l'équation 15 ; on y a figuré, en outre, la hauteur d'eau condensable réduite $w / \sin h$ ainsi que le coefficient de trouble d'Angström $\beta_{\mathrm{A}}$. La comparaison des valeurs fournies par les équations 13 et 15 montre que la vérification est bonne, les écarts étant, en général, inférieurs à $20 \%$.

\section{Conclusion.}

Nous avons établi des formules qui, d'une manière satisfaisante, permettent le calcul du rayonnement global horizontal et du rayonnement direct normal instantanés, par temps clair, pour le site de Carpentras, ainsi que les énergies cumulées sur une journée. Une équation a, d'autre part, été établie permettant l'évaluation du rayonnement direct à partir du global horizontal. Enfin, nous avons trouvé une relation simple entre le rayonnement diffus reçu sur le plan horizontal et le facteur de trouble de Linke.

Il est bien évident que les coefficients intervenant dans toutes ces équations relativement simples ne sont valables que pour le site étudié. Cependant, nous pensons que des équations de même type sont valables sur d'autres sites en France et qu'il serait intéressant de suivre les variations des coefficients d'une région à l'autre.

Ainsi, à Mâcon, le 14 juin 1976 (référence 1, page 111) on trouve, pour les équations 3 et 4 :

$$
B=967,64 \mathrm{~W} \cdot \mathrm{m}^{-2} \quad \beta=1,499 .
$$

A Carpentras, le 23 juin 1979,

$$
B=957,78 \mathrm{~W} \cdot \mathrm{m}^{-2} \quad \beta=1,361 .
$$

Les facteurs $B$ sont pratiquement les mêmes, alors que les $\beta$ diffèrent sensiblement.

Une étude plus exhaustive pourra être entreprise lorsque l'on disposera de données statistiques concernant le rayonnement direct en un nombre suffisant de stations météorologiques. L'extension du réseau de stations de relevés de données solaires semble d'ailleurs prouver la nécessité d'une analyse fine en chaque site particulier.

\section{Remerciements.}

Les auteurs expriment leur gratitude à Monsieur Coudert du Centre radiométrique de Carpentras (Météorologie Nationale) pour les renseignements qu'il a bien voulu leur communiquer.

\section{Appendice.}

Nomenclature des notations.

$\phi \quad$ latitude,

$\delta$ déclinaison solaire,

$h$ hauteur du soleil,

$\tau$ temps, en heures, compté à partir de midi, temps solaire vrai,

$G_{\mathrm{OH}}^{*}$ éclairement énergétique solaire global, par unité de surface, exprimé en W. $\mathrm{m}^{-2}$,

$I_{\mathrm{ON}}^{*}$ éclairement énergétique direct normal, par unité de surface, exprimé en $\mathrm{W} . \mathrm{m}^{-2}$,

$S S_{0}$ durée maximale d'ensoleillement en heures,

$\beta_{\mathrm{A}} \quad$ coefficient de trouble d'Angström,

$G_{\mathrm{OH}}$ énergie par unité de surface ou irradiation globale horizontale en Wh. $\mathrm{m}^{-2}$,

$T_{\mathrm{L}}$ facteur de trouble de Linke,

$J_{\mathbf{N}}^{*} \quad$ éclairement énergétique direct normal extraterrestre, par unité de surface en W. $\mathrm{m}^{-2}$,

$w$ hauteur d'eau condensable exprimée en $\mathrm{cm}$,

$D_{\mathrm{OH}}^{*} \quad$ éclairement énergétique diffus reçu sur un plan horizontal, par unité de surface, exprimé en W. $\mathrm{m}^{-2}$.

\section{Bibliographie}

[1] Schwartz, L. M., Louat, R. et Menguy, G., Revue Phys. Appl. 15 (1980) 103-112.

[2] Perrin de Brichambaut, C., Supplément aux Cahiers Afedes No 1, Juillet 1975, p. 23.

[3] Memosol, Mémento d'Héliotechnique (Editions européennes thermique et industrie (EETI)), 30 rue de la Source, 75016 Paris.

[4] Perrin de Brichambaut, C. et Vauge, C., Le gisement solaire (TEC et DOC) Paris 1982, p. 49. 